JOURNAL OF THE

AMERICAN MATHEMATICAL SOCIETY

Volume 18, Number 3, Pages 655-684

S 0894-0347(05)00481-9

Article electronically published on April 13, 2005

\title{
VALUATIONS AND MULTIPLIER IDEALS
}

\author{
CHARLES FAVRE AND MATTIAS JONSSON
}

\section{Contents}

Introduction

1. Background

2. Multiplier ideals of tree potentials

3. Multiplier ideals of ideals

4. Multiplier ideals of psh functions

5. Singularity exponents and Kiselman numbers

6. The ascending chain condition

7. Ideals as multiplier ideals

8. Equisingularity

Appendix A. Subadditivity and Skoda's Theorem

References

\section{INTRODUCTION}

This article is the third of a series of work on a new approach to the study of singularities of various objects in a local, two-dimensional setting. Our focus in the present paper is on multiplier ideals and singularity exponents.

In the discussion below, we fix an equicharacteristic zero, two-dimensional regular local ring $(R, \mathfrak{m})$ with algebraically closed residue field. An important example is the ring $R=\mathcal{O}_{0}$ of holomorphic germs at the origin in $\mathbf{C}^{2}$.

In [FJ1], we introduced the space $\mathcal{V}$ consisting of all $\mathbf{R}_{+} \cup\{+\infty\}$-valued valuations on $R$ centered at $\mathfrak{m}$, and normalized by $\nu(\mathfrak{m})=1$. This space is naturally a tree: it is a union of (uncountably many) real segments patched together in such a way that $\mathcal{V}$ remains homotopic to a point. It is also an $\mathbf{R}$-tree in the classical sense for a natural metric. We hence call $\mathcal{V}$ the valuative tree. It encodes in a natural way all possible blowups of $R$ centered at $\mathfrak{m}$ and therefore gives a way of measuring quite precisely singularities of different kinds of objects. The points in $\mathcal{V}$ that are not ends form the subtree $\mathcal{V}_{\mathrm{qm}}$ of quasimonomial valuations. These valuations, which can alternatively be characterized as Abhyankar valuations of rank 1 or as

Received by the editors January 16, 2004.

2000 Mathematics Subject Classification. Primary 14B05; Secondary 32U25, 13 H05.

Key words and phrases. Valuations, multiplier ideals, singularity exponents, Arnold multiplicity, Lelong numbers, Kiselman numbers, trees, Laplace operator.

The second author was partially supported by NSF Grant No. DMS-0200614.

(C)2005 American Mathematical Society Reverts to public domain 28 years from publication 
push-forwards of monomial valuations under birational morphisms, play a central role in our approach.

We used the valuative tree to study singularities of ideals $I \subset R$ in [FJ1, and of plurisubharmonic (psh) functions $u$ in FJ2 (in which case $R=\mathcal{O}_{0}$ ). We showed that $I$ and $u$ both give rise to functions $g_{I}$ and $g_{u}$ on $\mathcal{V}_{\mathrm{qm}}$, called the tree transforms of $I$ and $u$. These are defined as follows. For an ideal $I$ and $\nu \in \mathcal{V}_{\mathrm{qm}}$, we set $g_{I}(\nu):=$ $\nu(I)=\min \{\nu(\phi): \phi \in R\}$. For a psh function, the definition of $g_{u}(\nu)=\nu(u)$ is more involved but can be interpreted either as a generalized Lelong number in the sense of Demailly or as the push-forward of a Kiselman number under a birational morphism.

It turns out that the tree transforms $g_{I}$ and $g_{u}$ enjoy strong concavity properties. We denote by $\mathcal{P}$ the closure of the cone generated by the functions on $\mathcal{V}_{\mathrm{qm}}$ of the form $c g_{I}$ over all $c>0$ and all ideals $I$ (or equivalently by all $g_{u}$ 's for psh functions $u$ ). An element in $g \in \mathcal{P}$ is called a tree potential. Tree potentials can also be identified with positive measures on $\mathcal{V}$. In the case of an ideal, the measure is atomic and its decomposition into atoms is equivalent to Zariski's factorization of integrally closed ideals. In the case of a psh function, the measure determines the most important features of the singularity at the origin - a somewhat vague assertion that the paper at hand serves to substantiate. A general tree potential may be loosely viewed as a formal analogue of a psh function.

Multiplier ideals have emerged in recent years as a fundamental tool in algebraic and analytic geometry. We refer to the book $\mathrm{La}$ for a detailed account on this subject, on its range of applications, and for precise references. See also [D] for a more analytic point of view. Here we content ourselves with recalling a few definitions adapted to our setting.

Fix a psh function $u$ defined near the origin in $\mathbf{C}^{2}$. The multiplier ideal $\mathcal{J}(u)$ is the set of holomorphic germs $\phi \in \mathcal{O}_{0}$ such that $|\phi|^{2} \exp (-2 u)$ is locally integrable. As $u$ may take the value $-\infty, \mathcal{J}(u)$ is in general strictly included in $\mathcal{O}_{0}$. To worse behavior of $u$ at 0 , i.e., for $u$ decreasing faster to $-\infty$, correspond deeper ideals $\mathcal{J}(u)$. The multiplier ideal hence measures, in some sense, the degree of singularity of $u$.

To an ideal $I \subset R$ (or more generally a formal power $I^{c}, c>0$ ) we can also associate a multiplier ideal $\mathcal{J}(I)$. One way to do this uses resolution of singularities, whereas Lipman [Li] gives a more intrinsic definition. It is also possible to associate an asymptotic multiplier ideal to a graded system of ideals, i.e., a sequence $\left(I_{k}\right)_{k=1}^{\infty}$ of ideals in $R$ such that $I_{k} I_{l} \subset I_{k+l}$.

Our first objective is to show that all these multiplier ideals can be analyzed in a unified way using tree potentials. More precisely, to any tree potential $g$ on $\mathcal{V}_{\text {qm }}$ we associate a multiplier ideal $\mathcal{J}(g) \subset R$ as follows: an element $\psi \in R$ belongs to $\mathcal{J}(g)$ iff the function $\chi_{\psi, g}(\nu)=g(\nu) /(\nu(\psi)+A(\nu))$ on $\mathcal{V}_{\mathrm{qm}}$ is uniformly bounded by a constant $<1$. Here $A$ is a function on $\mathcal{V}_{\mathrm{qm}}$ called thinness. It contains information on the relative canonical divisor of suitable birational models of $\operatorname{Spec} R$, as in the classical algebraic definition of multiplier ideals (see Section 1.2.9 for details).

We then show that the multiplier ideal of an ideal, a psh function, or a graded system of ideals, coincides with that of the corresponding tree transform. Let us be more precise. If $I$ is an ideal and $c>0$, then we show that the multiplier ideal of $I^{c}$ coincides with that of the tree potential $c g_{I}$. Our proof essentially consists of translating classical conditions for defining multiplier ideals into our language. In 
the case of a graded system of ideals, the condition $I_{k} I_{l} \subset I_{k+l}$ implies a statement $g_{k+l} \leq g_{k}+g_{l}$ on the level of tree potentials $\left(g_{k}\right.$ is the tree potential of $\left.I_{k}\right)$. As we show, the sequence $k^{-1} g_{k}$ converges to a tree potential $g$ whose multiplier ideal coincides with that of the graded system.

Similarly, we prove that if $u$ is psh, then the multiplier ideals $\mathcal{J}\left(g_{u}\right)$ and $\mathcal{J}(u)$ coincide. The proof is now more involved. We first use Demailly's approximation technique to reduce to the case of a psh function with logarithmic singularities. The latter case is then reduced to the statement $\mathcal{J}\left(I^{c}\right)=\mathcal{J}\left(c g_{I}\right)$, proved earlier, for a suitable chosen ideal $I$ and $c>0$. Many of the arguments involved in the reductions draw from [DK], but our proof also depends heavily on the fact that the tree transform $u \mapsto g_{u}$ behaves well under Demailly approximation.

Apart from providing a unifying framework, tree potentials can be used as a powerful tool for studying many questions regarding multiplier ideals. As support to this claim, we give three applications of our approach.

The first two concern singularity exponents. To a general tree potential $g$ is associated a number $c(g)$, called the singularity exponent or log-canonical threshold of $g$. It is defined by $c(g)=\sup \{c>0: \mathcal{J}(c g)=R\}$. If $g=g_{u}$ is the tree transform of a psh function $u$, then the singularity exponent is given by $c(u)=\sup \{c>0$ : $\left.\exp (-2 c u) \in L_{\text {loc }}^{1}\right\}$ and is a number measuring the "strength" of the singularity of $u$ at the origin. Various bounds were known for $c(u)$ in terms of the Lelong number [S] and Kiselman numbers [Ki2] of $u$. We sharpen these bounds, and show that $c(u)$ can in fact be computed explicitly in terms of all Kiselman numbers of $u$. We also prove that for $c=c(u)$, the function $\exp (-2 c u)$ is never integrable. This provides an affirmative answer to the openness conjecture (in dimension 2) by Demailly and Kollár (see DK] Remark 5.3]). In fact, we establish the more precise estimate $\operatorname{Vol}\{u<\log r\} \gtrsim r^{2 c(u)}$ as $r \rightarrow 0$, and we improve a recent result by Blel and Mimouni [BM, Mi] by proving that if $u$ has Lelong number one, then $\exp (-2 u)$ fails to be locally integrable at the origin iff $d d^{c} u$ is the sum of the current of integration on a smooth curve and a current with zero Lelong number.

We then consider the set of complex singularity exponents $\mathbf{c}=\{c(\log |\psi|)\}$ when $\psi$ ranges over all holomorphic functions. Shokurov [S] used Mori's minimal program to show that $\mathbf{c}$ satisfies the so-called ascending chain condition: any increasing sequence in $\mathbf{c}$ is eventually stationary. Kuwata $[\mathrm{Ku}$ subsequently improved this result by characterizing explicitly all real numbers lying in c. In 2000, Phong-Sturm in PS] gave a completely analytic proof of this result. We present an algebraic proof in Section [6] independent of both approaches above. It is conjectured (see [DK] Remark 3.5]) that the ascending chain condition applies to the set of complex singularity exponents in any dimension (see $[\mathrm{MP}$ ] for the most recent result in this direction). We hope that our result might lead to further developments in higher dimensions.

As a third application, we prove that any integrally closed ideal in $R$ is the multiplier ideal of some formal power of an ideal. This result was recently proved, independently, by Lipman and Watanabe [LW].

Finally we prove quite generally that a tree potential is completely characterized by the collection of multiplier ideals $\{\mathcal{J}(t g)\}_{t \geq 0}$. As a consequence, two psh functions $u$ and $v$ have identical multiplier ideals $\mathcal{J}(t u)=\mathcal{J}(t v)$ for all $t \geq 0$ iff they are equisingular by which we mean that they have the same transforms $g_{u}=g_{v}$. Equisingularity may be geometrically interpreted as follows: for any composition $\pi$ 
of blowups, the Lelong numbers of the pull-backs $\pi^{*} u$ and $\pi^{*} v$ are the same at any point on the exceptional divisor $\pi^{-1}(0)$; see [FJ2, Proposition 6.2].

Since the results on singularity exponents for psh functions are arguably the most striking ones in the paper, we wish to briefly explain our approach, not using the language of valuations. Fix a psh function $u$ and assume, for simplicity, that $d d^{c} u$ does not charge any curve. To any irreducible (possibly singular) curve $D$ at the origin and any real number $t \geq 1$ we associate a family of punctured, conical regions $\mathcal{A}(r)$ of diameter $r$. Here $t$ determines the "thinness" of the region. See Figure 2 We have $\operatorname{Vol} \mathcal{A}(r) \sim r^{2 A}$ for some $A>0$, and in $\mathcal{A}(r)$ we have $u \leq \nu(u) \log r+O(1)$, for some (maximal) real number $\nu(u) \geq 0$. If $\exp (-2 c u)$ is locally integrable at the origin, it is integrable in $\mathcal{A}(r)$, which easily implies $c \nu(u)<A$. The crucial fact is the existence of an optimal region $\mathcal{A}(r)$ detecting integrability: for this region we have $c(u) \nu(u)=A$, which yields nonintegrability of $\exp (-2 c(u) u)$, i.e., the openness conjecture. Moreover, the curve $D$ associated to this region is smooth, and then $\nu(u)$ is a Kiselman number of $u$. It is to establish the existence and main properties of the optimal region $\mathcal{A}$ that we bring valuations into the picture. In particular, the concavity properties of the tree transform of $u$ play a key role.

Most results presented here are probably not particular to dimension 2 . The main difficulty in extending our approach to higher dimensions lies in understanding the analogue of the valuative tree. We hope to tackle this problem in future research.

The present article relies in an essential way on the analysis and formalism in our previous work: we recall in Section 1 the main results of [FJ1, FJ2 that will be used. The rest of the paper is then organized as follows: in Section 2 we give the definition and main properties of multiplier ideals of tree potentials. As we show in Sections 3 and 4 , this notion naturally generalizes multiplier ideals of formal powers of ideals, graded systems of ideals, and psh functions. Sections 5 6 and 7 contain the applications mentioned above. We study equisingularity in Section 8 and end the paper with an appendix containing two proofs.

\section{BACKGROUND}

In this section we give a brief review of the valuative tree and its applications to the study of ideals and plurisubharmonic (psh) functions. For details, we refer to [FJ1] for Sections 1.1 to [1.3, and to [FJ2] for Section 1.4.

1.1. Conventions. In general, $(R, \mathfrak{m})$ denotes an equicharacteristic zero, twodimensional regular local ring with algebraically closed residue field $k$. We will refer to this as the general case.

Whenever we talk about psh functions, we will always be in the analytic case, meaning that $R=\mathcal{O}_{0}$ is the ring of holomorphic germs at the origin in $\mathbf{C}^{2}$. Then $k=\mathbf{C}$ and $\mathfrak{m}$ is the maximal ideal of germs vanishing at the origin.

In general we write $(\hat{R}, \hat{\mathfrak{m}})$ for the completion of $R$. It is the ring of formal power series in two variables with coefficients in $k$.

1.2. The valuative tree. Our starting point is the approach to valuations worked out in [FJ1].

1.2.1. Valuations ([FJ1, Section 1.2]). We consider the space $\mathcal{V}$ of centered, normalized valuations on $R$, i.e., the set of functions $\nu: R \rightarrow[0, \infty]$ satisfying: 
(i) $\nu\left(\psi \psi^{\prime}\right)=\nu(\psi)+\nu\left(\psi^{\prime}\right)$ for all $\psi, \psi^{\prime}$;

(ii) $\nu\left(\psi+\psi^{\prime}\right) \geq \min \left\{\nu(\psi), \nu\left(\psi^{\prime}\right)\right\}$ for all $\psi, \psi^{\prime}$;

(iii) $\nu(0)=\infty,\left.\nu\right|_{\mathbf{C}^{*}}=0, \nu(\mathfrak{m}):=\min \{\nu(\psi) ; \psi \in \mathfrak{m}\}=1$.

Then $\mathcal{V}$ is equipped with a natural partial ordering: $\nu \leq \mu$ iff $\nu(\psi) \leq \mu(\psi)$ for all $\psi \in \mathfrak{m}$. The multiplicity valuation $\nu_{\mathfrak{m}}$ defined by $\nu_{\mathfrak{m}}(\psi)=m(\psi)=\max \left\{k ; \psi \in \mathfrak{m}^{k}\right\}$ is the unique minimal element of $\mathcal{V}$. (See [FJ1, Section 1.5.1].)

Any valuation on $R$ extends uniquely to a valuation in its completion $\hat{R}$; hence the valuation spaces attached to $R$ and $\hat{R}$ are isomorphic. (See [Sp, Theorem 3.1].)

1.2.2. Curve valuations ([FJ1, Section 1.5.5]). Some natural maximal elements are the curve valuations defined as follows. To each irreducible (possibly formal) curve $C$ we associate $\nu_{C} \in \mathcal{V}$ defined by $\nu_{C}(\psi)=C \cdot\{\psi=0\} / m(C)$, where "." denotes intersection multiplicity and $m$ multiplicity. If $C$ is defined by $\phi \in \hat{\mathfrak{m}}$, then we also write $\nu_{C}=\nu_{\phi}$. Note that $\nu_{\phi}(\phi)=\infty$.

The set $\mathcal{C}$ of local irreducible curves carries a natural (ultra)metric in which $\mathcal{C}$ has diameter 1 . It is given by $d_{\mathcal{C}}(C, D)=m(C) m(D) / C \cdot D$. (See [FJ1, Lemma 3.56].)

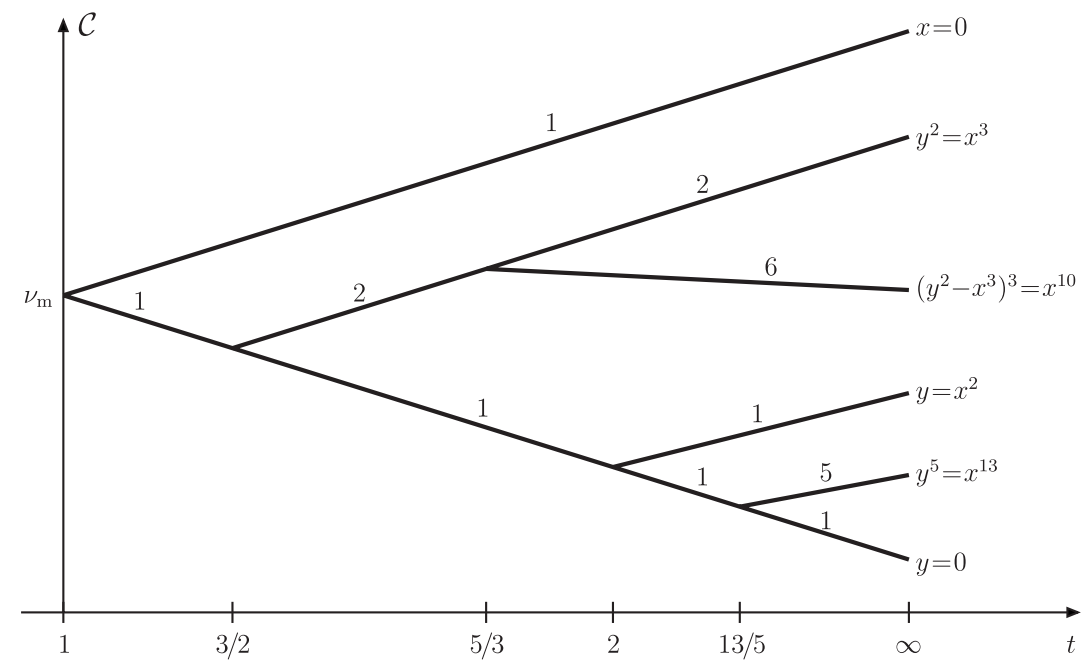

Figure 1. The valuative tree. The segments consist of valuations of the form $\nu_{\phi, t}$, where $\phi=x, y^{2}-x^{3}, \ldots, y$ and the skewness parameter $t$ ranges from 1 to $\infty$. Skewness $t=1$ gives the multiplicity valuation $\nu_{\mathfrak{m}}$ and skewness $t=\infty$ the curve valuation $\nu_{\phi}$. The integer label above a segment indicates multiplicity (Section 1.2.6.

1.2.3. Quasimonomial valuations ([FJ1, Section 1.5.4]). Arguably the most important valuations in $\mathcal{V}$ are the quasimonomial ones 1 They are of the form $\nu_{C, t}$, where

\footnotetext{
${ }^{1} \mathrm{~A}$ quasimonomial valuation can be made monomial (i.e., completely determined by its values on a pair of local coordinates $(x, y))$ by a birational morphism. Quasimonomial valuations are also known as Abhyankar valuations of rank 1; see ELS.
} 
$C \in \mathcal{C}$ and $t \in[1, \infty)$, and satisfy $\nu_{C, t}(\psi)=\min \left\{\nu_{D}(\psi): d_{\mathcal{C}}(C, D) \leq t^{-1}\right\}$. We have $\nu_{C, s}=\nu_{D, t}$ iff $s=t \geq d_{\mathcal{C}}(C, D)^{-1}$. (See [FJ1, Proposition 3.55].) Thus $\mathcal{V}_{\text {qm }}$, the set of all quasimonomial valuations, is naturally a quotient of $\mathcal{C} \times[1, \infty)$ and has a natural tree structure: if $\nu, \nu^{\prime} \in \mathcal{V}_{\mathrm{qm}}$ and $\nu<\nu^{\prime}$, then the segment $\left[\nu, \nu^{\prime}\right]=\left\{\mu \in \mathcal{V}_{\mathrm{qm}}: \nu \leq \mu \leq \nu^{\prime}\right\}$ is isomorphic to a compact real interval. See [FJ1, Theorem 3.57] and Figure 1. We set $\nu_{\phi, t}:=\nu_{C, t}$ when $C=\{\phi=0\}$.

Quasimonomial valuations are of two types: divisorial and irrational, depending on whether the parameter $t$ is rational or irrational 2 We write $\mathcal{V}_{\text {div }}$ for the set of divisorial valuations.

The full space $\mathcal{V}$ is the completion of $\mathcal{V}_{\mathrm{qm}}$ in the sense that every element in $\mathcal{V}$ is the limit of an increasing sequence in $\mathcal{V}_{\mathrm{qm}}$. It is hence also naturally a tree, called the valuative tree. The ends of $\mathcal{V}$ are exactly the elements of $\mathcal{V} \backslash \mathcal{V}_{\mathrm{qm}}$ and are either curve valuations or infinitely singular valuations 3

1.2.4. Skewness and intersection multiplicity ([FJ1, Section 3.3]). An important invariant of a valuation is its skewness $\alpha$ defined by $\alpha(\nu)=\sup \{\nu(\phi) / m(\phi): \phi \in \mathfrak{m}\}$. Skewness naturally parameterizes the trees $\mathcal{V}_{\mathrm{qm}}$ and $\mathcal{V}$ in the sense that $\alpha: \mathcal{V}_{\mathrm{qm}} \rightarrow$ $[1, \infty)$ is strictly increasing and restricts to a bijection onto its image on any segment; indeed $\alpha\left(\nu_{\phi, t}\right)=t$ for any $\nu_{\phi, t} \in \mathcal{V}_{\mathrm{qm}}$. Thus divisorial (irrational) valuations have rational (irrational) skewness. Curve valuations have infinite skewness whereas the skewness of an infinitely singular valuation may or may not be finite.

The tree structure on $\mathcal{V}$ implies that any collection $\left(\nu_{i}\right)_{i \in I}$ of valuations in $\mathcal{V}$ admits an infimum $\wedge_{i} \nu_{i}$; see [FJ1, Corollary 3.15]. Together with skewness, this allows us to define an intersection product on $\mathcal{V}$ : we set $\nu \cdot \mu:=\alpha(\nu \wedge \mu) \in[1, \infty]$. This is a normalized extension of the intersection product on $\mathcal{C}$ as $C \cdot D=\left(\nu_{C}\right.$. $\left.\nu_{D}\right) m(C) m(D)$. If $\nu \in \mathcal{V}$ and $\phi \in \mathfrak{m}$ is irreducible, then $\nu(\phi)=m(\phi)\left(\nu \cdot \nu_{\phi}\right)$. Moreover, if $\nu(\phi)$ is irrational, then $\nu=\nu_{\phi, t}$ with $t=\nu(\phi) / m(\phi)$.

1.2.5. Tangent space and weak topology ([FJ1, Sections 3.1-3.2]). Let $\mu$ be a valuation in $\mathcal{V}$. Declare $\nu, \nu^{\prime} \in \mathcal{V} \backslash\{\mu\}$ to be equivalent if the segments $\left.] \mu, \nu\right]$ and ]$\left.\mu, \nu^{\prime}\right]$ intersect. An equivalence class is called a tangent vector at $\mu$ and the set of tangent vectors at $\mu$, the tangent space, is denoted by $T \mu$. If $\vec{v}$ is a tangent vector, we denote by $U(\vec{v})$ the set of points in $\mathcal{V}$ defining the equivalence class $\vec{v}$. The points in $U(\vec{v})$ are said to represent $\vec{v}$.

A point $\mu$ in the tree $\mathcal{V}$ is an end, a regular point, or a branch point when $T \mu$ contains one, two, or three or more points, respectively. In terms of valuations: the ends of $\mathcal{V}$ are curve and infinitely singular valuations; the regular points are irrational valuations; and the branch points are divisorial valuations, at which the tangent space is in bijection with the complex projective line $\mathbf{P}^{1}$ and hence uncountable. See [FJ1, Theorem 3.20].

We endow $\mathcal{V}$ with the weak topology, generated by the sets $U(\vec{v})$ over all tangent vectors $\vec{v}$; this turns $\mathcal{V}$ into a compact (Hausdorff) space. If $\nu_{k} \rightarrow \nu$, then $\nu_{k} \wedge \mu \rightarrow$ $\nu_{k} \wedge \mu$ for all $\mu \in \mathcal{V}$. The weak topology on $\mathcal{V}$ is characterized by $\nu_{k} \rightarrow \nu$ iff $\nu_{k}(\phi) \rightarrow \nu(\phi)$ for all $\phi \in R$; see [FJ1 Theorem 5.1].

\footnotetext{
${ }^{2}$ A quasimonomial valuation $\nu$ is irrational iff $\nu(R) \not \subset \mathbf{Q}$, hence the name.

${ }^{3}$ The latter are represented by infinite Puiseux series whose exponents are rational numbers with unbounded denominators.
} 
1.2.6. Multiplicities ([FJ1, Section 3.4]). By setting $m(\nu):=\min \{m(C): C \in$ $\left.\mathcal{C}, \nu_{C} \geq \nu\right\}$ we extend the notion of multiplicity from $\mathcal{C}$ to $\mathcal{V}_{\mathrm{qm}}$. Clearly $m$ : $\mathcal{V}_{\mathrm{qm}} \rightarrow \mathbf{N}$ is increasing and hence extends to all of $\mathcal{V}$. In fact $m(\nu)$ divides $m(\mu)$ whenever $\nu \leq \mu$. The infinitely singular valuations are characterized as having infinite multiplicity.

As $m$ is increasing and integer-valued, it is piecewise constant on any segment $\left[\nu_{\mathfrak{m}}, \nu_{\phi}\right]$, where $\phi \in \mathcal{C}$. This implies that $m(\vec{v})$ is naturally defined for any tangent vector $\vec{v}$. If $\nu$ is nondivisorial, then $m(\vec{v})=m(\nu)$ for any $\vec{v} \in T \nu$.

If $\nu$ is divisorial, then the situation is more complicated. Suffice it to say that there exists an integer $b(\nu)$, divisible by $m(\nu)$, such that $m(\vec{v})=b(\nu)$ for all but at most two tangent vectors $\vec{v}$ at $\nu$. We call $b(\nu)$ the generic multiplicity of $\nu$.

1.2.7. Approximating sequences ([FJ1, Section 3.5]). Consider a quasimonomial valuation $\nu \in \mathcal{V}_{\mathrm{qm}}$. The multiplicity $m$ is integer-valued and piecewise constant on the segment $\left[\nu_{\mathfrak{m}}, \nu\right]$, hence has a finite number $g$ (possibly zero) of jumps. Thus there are divisorial valuations $\nu_{i}, 0 \leq i \leq g$ and integers $m_{i}$, such that

$$
\nu_{\mathfrak{m}}=\nu_{0}<\nu_{1}<\cdots<\nu_{g}<\nu_{g+1}=\nu
$$

and $m(\mu)=m_{i}$ for $\left.\left.\mu \in\right] \nu_{i}, \nu_{i+1}\right], 0 \leq i \leq g$. We call the sequence $\left(\nu_{i}\right)_{i=1}^{g}$ the approximating sequence associated to $\nu$. It plays a prominent role in $[\mathrm{Sp}]$.

The concept of approximating sequences extends naturally to valuations that are not quasimonomial: for curve valuations the sequences are still finite, for infinitely singular valuations they are infinite.

1.2.8. Thinness ([FJ1, Section 3.6]). Skewness $\alpha$ is a parameterization of $\mathcal{V}$ that does not "see" multiplicities. Another parameterization, of crucial importance, is thinness $A$, defined as follows. If $\nu \in \mathcal{V}_{\mathrm{qm}}$, then

$$
A(\nu)=2+\int_{\nu_{\mathrm{m}}}^{\nu} m(\mu) d \alpha(\mu)
$$

In terms of (1.1) we have $A(\nu)=2+\sum_{0}^{g} m_{i}\left(\alpha_{i+1}-\alpha_{i}\right)$ with $\alpha_{i}=\alpha\left(\nu_{i}\right)$. Note that $A(\nu) \leq 1+m(\nu) \alpha(\nu)$. Just like skewness, we may define $A(\nu)$ also for $\nu \notin \mathcal{V}_{\mathrm{qm}}$.

The inequality $A(\nu) \geq 1+\alpha(\nu)$ always holds, with equality iff $m(\nu)=1$. Moreover, $A(\nu)<m(\nu) \alpha(\nu)$ as soon as $m(\nu)>1$, and $A(\nu)-m(\nu) \alpha(\nu) \rightarrow-\infty$ as $\nu$ increases towards an infinitely singular valuation.

1.2.9. Geometric interpretation of divisorial valuations ([FJ1, Chapter 6]). Every divisorial valuation $\nu$ arises as follows: there exists a finite composition of point blowups $\pi: X \rightarrow \operatorname{Spec} R$ (i.e., $\pi: X \rightarrow\left(\mathbf{C}^{2}, 0\right)$ in the analytic case) and an exceptional component $E$ (i.e., an irreducible component of $\pi^{-1}(0)$ ) such that $\nu=b^{-1} \pi_{*} \operatorname{div}_{E}$, where $b=b(\nu)$ is the generic multiplicity at $\nu$ and $\operatorname{div}_{E}$ denotes the order of vanishing along $E$. The generic multiplicity of $\nu$ is then equal to the multiplicity of any curvette of $\nu$, i.e., the image by $\pi$ of any smooth curve intersecting $E$ transversely at a smooth point. In fact, every generic tangent vector at $\nu$ is represented by the curve valuation associated to a curvette [FJ1, Section 6.6.1]. We also have $A(\nu)=a / b$, where $a-1$ is equal to the order of vanishing along $E$ of the Jacobian determinant of $\pi$ FJ1, Theorem 6.22]. 
1.2.10. Borel measures and tree potentials ([FJ1. Chapter 7] or [FJ2 Section 5]). Let $\mathcal{M}$ be the space of (weak, regular) positive Borel measures on $\mathcal{V} 4$ endowed with the topology of vague convergence. We will identify $\nu \in \mathcal{V}$ with its Dirac mass $\delta_{\nu} \in \mathcal{M}$, thus viewing $\mathcal{V}$ as a (compact) subset of $\mathcal{M}$.

Any $\rho \in \mathcal{M}$ determines a real-valued function $g_{\rho}$ on $\mathcal{V}_{\mathrm{qm}}$ defined by $g_{\rho}(\nu)=$ $\int_{\mathcal{V}} \mu \cdot \nu d \rho(\mu)$. A function of the form $g_{\rho}$ is called a tree potential. The space $\mathcal{P}$ of tree potentials is a closed convex cone in $\mathcal{V}_{\mathrm{qm}}^{\mathbf{R}}$ (under pointwise convergence) and the map $\rho \mapsto g_{\rho}$ is a homeomorphism of $\mathcal{M}$ onto $\mathcal{P}$ whose inverse naturally defines a Laplace operator $\Delta: \mathcal{P} \rightarrow \mathcal{M} 5$ The mass of $\Delta g$ is $g\left(\nu_{\mathfrak{m}}\right)$. See [FJ1. Theorem 7.64].

If $g \in \mathcal{P}$, then $g$ is increasing and concave on any segment $\left[\nu_{\mathfrak{m}}, \nu_{0}\right]$ (parameterized by skewness) in $\mathcal{V}$. If $\nu \in] \nu_{\mathfrak{m}}, \nu_{0}$ [, then the left derivative of $g$ at $\nu$ with respect to skewness is equal to $\rho\{\mu \geq \nu\}$, whereas the right derivative is given by $\rho(U(\vec{v}))$, where $\rho=\Delta g$ and $\vec{v}$ denotes the tangent vector at $\nu$ represented by $\nu_{0}$. We always have $\rho\{\mu \geq \nu\} \alpha(\nu) \leq g(\nu) \leq g\left(\nu_{\mathfrak{m}}\right) \alpha(\nu)$ with equality (in either inequality) iff $\rho=\Delta g$ is supported on $\{\mu \geq \nu\}$.

A subtree of $\mathcal{V}$ is a subset $\mathcal{T}$ such that $\nu \in \mathcal{T}$ and $\mu \leq \nu$ implies $\mu \in \mathcal{T}$. A subtree is finite if it has finitely many ends. If $g \in \mathcal{P}$ and $\mathcal{T}$ is a subtree of $\mathcal{V}$, then $g_{\mathcal{T}}$ denotes the infimum of all tree potentials coinciding with $g$ on $\mathcal{T}$.

The support of $g \in \mathcal{P}$ is the smallest subtree $\mathcal{T}$ for which $g=g_{\mathcal{T}}$. Alternatively, it is the smallest subtree containing supp $\Delta g$. The support of any tree potential is included in the closure of a countable union of finite trees.

1.3. Ideals ([FJ1, Section 8.1]). An ideal $I$ in $R$ is primary if $\mathfrak{m}^{n} \subset I$ for some $n>$ 0 . The integral closure of $I$ is the ideal $\bar{I}$ of $\phi \in R$ such that $\phi^{n}+a_{1} \phi^{n-1}+\cdots+a_{n}=0$ for some $n \geq 1$ and $a_{i} \in I^{i}$. If $I=\bar{I}$, then $I$ is integrally closed.

Any ideal $I$ in $R$ has an associated tree transform $g_{I}$, defined by $g_{I}(\nu)=\nu(I)=$ $\min \{\nu(\phi): \phi \in R\}$. This function $g_{I}$ belongs to $\mathcal{P}$, and thus defines a tree measure $\rho_{I}=\Delta g_{I} \in \mathcal{M}$. The latter measure has mass $m(I):=\nu_{\mathfrak{m}}(I)$.

If $I=\phi R$ is principal, then we write $g_{\phi}=g_{I}$ and $\rho_{\phi}=\rho_{I}$ : the latter measure is given by $\rho_{\phi}=\sum_{i} n_{i} m_{i} \nu_{i}$, where $\phi=\prod \phi_{i}^{n_{i}}$ is the factorization of $\phi$ into irreducible factors, $m_{i}=m\left(\phi_{i}\right)$ and $\nu_{i}$ is the curve valuation associated to $\phi_{i}$.

If $I$ is primary, then $\rho_{I}=\sum_{i} n_{i} b_{i} \nu_{i}$, where $n_{i} \in \mathbf{N}$ and $\nu_{i}$ are divisorial valuations with generic multiplicity $b_{i}$. The valuations $\nu_{i}$ are exactly the Rees valuations of $I$.

A general ideal $I$ is the product of a principal ideal and a primary ideal, hence has a tree measure of the form $\rho_{I}=\sum_{i} n_{i} b_{i} \nu_{i}+\sum_{i} n_{i} m_{i} \nu_{i}$.

To any measure $\rho$ is associated an ideal $I_{\rho}=\left\{\phi \in R: g_{\phi} \geq g_{\rho}\right\}$. When $\rho$ is of the previous form, then $\rho_{I_{\rho}}=\rho$, whereas $I_{\rho_{I}}$ is the integral closure of $I$. The decomposition of $\rho_{I}$ above gives an interpretation of Zariski's factorization theorem: if $I$ is integrally closed, then $I=\prod_{i} I_{\nu_{i}}^{n_{i}}$. If furthermore $I$ is primary, i.e., all the $\nu_{i}$ are divisorial, then $I=\bigcap_{i}\left\{\phi \in R: g_{\phi}\left(\nu_{i}\right) \geq g_{\rho}\left(\nu_{i}\right)\right\}$.

In general, the mass of $\rho_{I}$ on a curve valuation $\nu_{\phi}$ is the product of $m(\phi)$ and $\operatorname{div}_{\phi}(I):=\max \left\{k: \phi^{k} \mid \psi\right.$ for all $\left.\psi \in I\right\}$.

1.4. Psh functions. All plurisubharmonic (psh) functions are defined near the origin in $\mathbf{C}^{2}$. A psh function $u$ is said to have logarithmic singularities if there exist $c>0$ and holomorphic functions $\phi_{i} \in R$ such that $u=\frac{c}{2} \log \sum_{1}^{n}\left|\phi_{i}\right|^{2}+O(1)$.

\footnotetext{
${ }^{4}$ In [FJ1], $\mathcal{M}$ is denoted by $\mathcal{M}^{+}$.

${ }^{5}$ In [FJ1] $\mathcal{P}$ is denoted by $\mathcal{P}^{+}$, and tree potentials are called positive tree potentials.
} 
1.4.1. Lelong and Kiselman numbers. For a fixed choice of coordinates $(x, y)$, and weights $a, b>0$ the Kiselman number [Ki1, Ki2] of $u$ is defined to be

$$
\nu_{a, b}^{x, y}(u)=\lim _{r \rightarrow 0} \frac{a b}{\log r} \sup \left\{u:|x|<r^{1 / a},|y|<r^{1 / b}\right\} .
$$

We have $\nu_{\lambda a, \lambda b}^{x, y}(u)=\lambda \nu_{a, b}^{x, y}(u)$ for any $\lambda>0$. When $a=b=1$, the Kiselman number reduces to the Lelong number $\nu^{\mathrm{L}}(u)$. The latter does not depend on the choice of $(x, y)$.
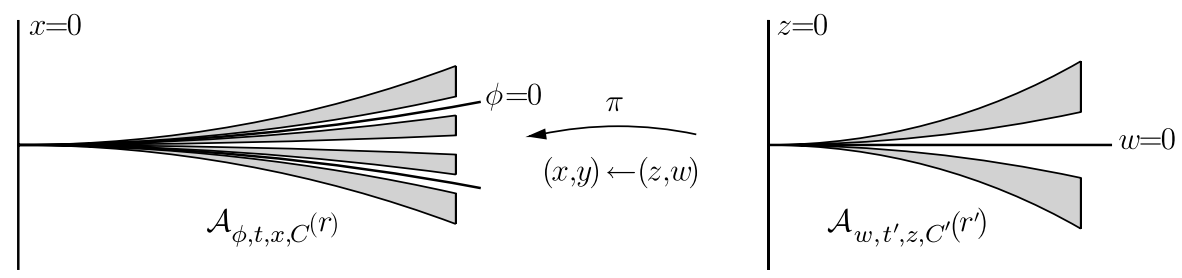

Figure 2. Characteristic regions, see Section 1.4.2 To the left is a characteristic region $\mathcal{A}$ around a singular curve $\{\phi=0\}$. If $\pi: X \rightarrow\left(\mathbf{C}^{2}, 0\right)$ desingularizes this curve, the preimage of $\mathcal{A}$ is a characteristic region around a smooth curve.

1.4.2. Evaluating valuations on psh functions ([FJ2, Section 3]). One can evaluate a quasimonomial valuation on a psh function as the normalized push-forward of a Kiselman number under a birational morphism. Concretely, this goes as follows. If $\nu \in \mathcal{V}_{\text {qm }}$ is quasimonomial, write $\nu=\nu_{\phi, t}$ for $\phi \in \mathfrak{m}$ irreducible with $m(\phi)=$ $m(\nu)=: m$. Pick a coordinate $x$ transverse to $\phi$, i.e., $x \in \mathfrak{m}$ is irreducible, $m(x)=1$ and $\nu_{x} \wedge \nu_{\phi}=\nu_{\mathfrak{m}}$. Also pick constants $0<C_{1}<C_{2}<\infty$ and consider the region

$$
\mathcal{A}(r)=\mathcal{A}_{\phi, t, x, C}(r):=\left\{|x|<r: C_{1}|x|^{m t}<|\phi|<C_{2}|x|^{m t}\right\}
$$

for small $r$. See Figure 2 We have $\operatorname{Vol} \mathcal{A}(r) \simeq r^{2 A}$, where $A=A(\nu)$ is the thinness of $\nu$. The value of $\nu$ on a psh function $u$ is then given by

$$
\nu(u)=\lim _{r \rightarrow 0} \frac{1}{\log r} \sup _{\mathcal{A}(r)} u ;
$$

this does not depend on the choices of $\phi, x$ or $C$. We always have the upper bound $u(q) \leq \nu(u) \log \|q\|+O(1)$ in $\mathcal{A}(r)$. If $u=\frac{c}{2} \log \sum_{1}^{n}\left|\phi_{i}\right|^{2}+O(1)$ has logarithmic singularities, then $\nu(u)=c \min _{i} \nu\left(\phi_{i}\right)=c \nu(I)$, where $I$ is the ideal generated by the $\phi_{i}$ 's. In this case we also have the lower bound $u(q) \geq \nu(u) \log \|q\|+O(1)$ for suitable choices of $C_{1}$ and $C_{2}$. See [FJ2, Proposition 4.3].

If $\nu \in \mathcal{V}_{\mathrm{qm}}$ is monomial, i.e., of multiplicity one, then there exist local coordinates $(x, y)$ such that $\nu=\nu_{y, t}$, where $t=\alpha(\nu) \geq 1$. In this case $\nu(u)$ equals the Kiselman number $\nu_{t, 1}^{x, y}(u)$. In particular $\nu_{\mathfrak{m}}(u)$ is the Lelong number of $u$.

1.4.3. Geometric interpretation. The value of divisorial valuations on psh functions can be interpreted along the lines of Section [1.2.9. Using the notation of that section, $b(\nu) \nu(u)$ is the Lelong number of the pull-back $\pi^{*} u$ at a generic point $p \in E$ [FJ2, Proposition 4.1]. Moreover, if $p \in E$ is a smooth point on $\pi^{-1}(0)$, then the Lelong number of the strict transform of $u$ under $\pi$ at $p$ is bounded from above by $b(\nu)^{-1} \rho_{u}(U(\vec{v}))$ [FJ2, Lemma 7.6]. Here $\rho_{u}$ is the tree measure of $u$ (see below) 
and $\vec{v}=\vec{v}_{p}$ is the tangent vector at $\nu$ associated to $p$; that is, $\vec{v}$ is represented by any curve valuation $\nu_{C}$ such that the strict transform of $C$ is smooth and intersects $E$ transversely at $p$.

1.4.4. Demailly approximation. Demailly devised a method, crucially important to our analysis, of approximating a general psh function $u$ with a sequence $u_{n}$ of psh functions with logarithmic singularities. This goes as follows: see Theorem 4.2 and its proof in $[\mathrm{DK}$ for details. Suppose $u$ is psh on a fixed ball $B$ containing the origin. For $n>0$ let $\mathcal{H}_{n}$ be the Hilbert space of holomorphic functions $\psi$ on $B$ such that $\int_{B}|\psi|^{2} e^{-2 n u}<\infty$. Let $\left(g_{n l}\right)_{1}^{\infty}$ be an orthonormal basis for $\mathcal{H}_{n}$ and set $u_{n}=\frac{1}{2 n} \log \sum_{l}\left|g_{n l}\right|^{2}$. If $0 \ni B^{\prime} \Subset B$ is a smaller ball, then there exists $k=k\left(u, n, B^{\prime}\right)<\infty$ such that $u_{n}-\frac{1}{2 n} \log \sum_{1}^{k}\left|g_{n l}\right|^{2}$ is bounded in $B^{\prime}$; in particular $u_{n}$ has a logarithmic singularity at the origin.

Demailly approximation interacts very well with the evaluation of quasimonomial valuations on psh functions: we have $0 \leq \nu(u)-\nu\left(u_{n}\right) \leq A(\nu) / n$, where $A$ denotes thinness [FJ2, Proposition 3.12].

1.4.5. Tree transforms ([FJ2 Section 6.1]). Any psh function $u$ has an associated tree transform $g_{u} \in \mathcal{P}$, defined by $g_{u}(\nu)=\nu(u)$ for $\nu \in \mathcal{V}_{\mathrm{qm}}$, as well as an associated tree measure $\rho_{u}=\Delta g_{u}$ on $\mathcal{V}$. That these are well defined follows from Demailly approximation. If $u$ is psh, then $\rho_{u}$ cannot put mass on a formal (i.e., non-analytic) curve valuation. When $\nu_{C}$ is a curve valuation associated to an analytic curve $C=\{\phi=0\}$, the mass of $\rho_{u}$ on $\nu_{C}$ is exactly $m(C)$ times the mass of the positive closed $(1,1)$ current $d d^{c} u$ on $C$. By Siu's theorem, this is equivalent to saying that $u=u^{\prime}+c \log |\phi|$ with $c=m(C)^{-1} \rho_{u}\left\{\nu_{\phi}\right\}$ and $u^{\prime}$ a psh function with $\rho_{u^{\prime}}\left\{\nu_{C}\right\}=0$.

\section{Multiplier ideals of tree Potentials}

In this section we define multiplier ideals of tree potentials and examine their main properties. As we will show in subsequent sections, this notion contains all previously known (to the authors) definitions of multiplier ideals, in a local, twodimensional setting. We also introduce some related singularity exponents.

Definition 2.1. Let $h: \mathcal{V}_{\mathrm{qm}} \rightarrow[0, \infty)$ be a tree potential on $\mathcal{V}$, i.e., $h \in \mathcal{P}$. We define the multiplier ideal $\mathcal{J}(h)$ of $h$ to be the ideal of elements $\psi \in R$ such that

$$
\sup _{\nu \in \mathcal{V}_{\mathrm{qm}}} \frac{h(\nu)}{\nu(\psi)+A(\nu)}<1 \text {. }
$$

Definition 2.2. Let $h \in \mathcal{P}$. The singularity exponent, or log-canonical threshold, of $h$ is the number $c(h):=\sup \{c>0: \mathcal{J}(c h)=R\}$. The Arnold multiplicity is the number $\lambda(h)=c(h)^{-1}$. Thus we have

$$
\lambda(h)=\sup _{\nu \in \mathcal{V}_{\mathrm{qm}}} \frac{h(\nu)}{A(\nu)} \quad \text { and } \quad c(h)=\inf _{\nu \in \mathcal{V}_{\mathrm{qm}}} \frac{A(\nu)}{h(\nu)} .
$$

Remark 2.3. It follows immediately from the definition that $\mathcal{J}(c(h) h) \subsetneq R$.

It will be useful to have a less elegant but more concrete criterion for membership in the multiplier ideal.

Proposition 2.4. Fix a tree potential $h \in \mathcal{P}$ and $\psi \in R$. Let $\rho_{h}$ and $\rho_{\psi}$ be the associated measures on $\mathcal{V}$. Then $\psi \in \mathcal{J}(h)$ iff the following hold:

(a) $\nu(\psi)+A(\nu)>h(\nu)$ for all quasimonomial valuations $\nu$; 
(b) $\rho_{\psi}\{\nu\}+m(\nu)>\rho_{h}\{\nu\}$ for all curve valuations $\nu$.

Moreover, we have

(i) if $\psi \notin \mathfrak{m}$, then it suffices to check (a)-(b) for $\nu$ of multiplicity one;

(ii) if $h=c g_{I}$ for some ideal I and $c>0$, then it suffices to check (a)-(b) for $\nu$ in the approximating sequence of some valuation in the support of $\rho_{I}$.

The proof is given below. Note that (2.1) is not a trivial consequence of (a).

2.1. Properties. Let us now state some basic properties of multiplier ideals associated to tree potentials. The proofs are given below. However, two of them are relegated to the appendix as they do not follow directly from our approach, but rely on the corresponding statements for ideals already proved in the literature.

Proposition 2.5. The multiplier ideal $\mathcal{J}(h)$ is integrally closed.

It is clear from the definition that if $g \leq h$, then $\mathcal{J}(h) \subset \mathcal{J}(g)$. A deeper result is the following semicontinuity statement.

Proposition 2.6. If $\left(h_{n}\right)_{1}^{\infty}$ is a decreasing sequence of tree potentials converging in $\mathcal{P}$ (i.e., pointwise) to a tree potential $h$, then $\mathcal{J}\left(h_{n}\right)=\mathcal{J}(h)$ for $n \gg 1$.

Notice that since $R$ is Noetherian, the sequence $\left(\mathcal{J}\left(h_{n}\right)\right)_{1}^{\infty}$ is stationary. This does not, however, immediately imply the proposition.

We can bound the Arnold multiplicity as follows.

Proposition 2.7. Consider $h \in \mathcal{P}$ with associated measure $\rho=\Delta h$. Then:

(i) $\frac{1}{2} h\left(\nu_{\mathfrak{m}}\right) \leq \lambda(h) \leq h\left(\nu_{\mathfrak{m}}\right)$;

(ii) $\lambda(h)>\frac{1}{2} h\left(\nu_{\mathfrak{m}}\right)$ iff there exists a tangent vector $\vec{v}$ at $\nu_{\mathfrak{m}}$ with $\rho U(\vec{v})>\frac{1}{2}$;

(iii) $\lambda(h)=h\left(\nu_{\mathfrak{m}}\right)$ iff $\rho$ is a point mass at a curve valuation of multiplicity one.

Next we have the following subadditivity property.

Proposition 2.8. If $h_{1}, h_{2} \in \mathcal{P}$, then $\mathcal{J}\left(h_{1}+h_{2}\right) \leq \mathcal{J}\left(h_{1}\right) \mathcal{J}\left(h_{2}\right)$.

Our proof uses a reduction to the case when the $h_{i}$ 's are proportional to tree transforms of ideals and is given in the appendix. The same holds for the following version of Skoda's Theorem:

Proposition 2.9. Let $\nu_{0}$ be a divisorial valuation with associated (simple complete) ideal $I_{0}=I_{\nu_{0}}$ and tree transform $g_{0}=g_{I_{0}}$. Then we have

$$
\mathcal{J}\left(h+2 g_{0}\right)=I_{0} \mathcal{J}\left(h+g_{0}\right)
$$

for any tree potential $h \in \mathcal{P}$.

On the other hand, Proposition 2.10 is a version of Skoda's Theorem that we can prove directly.

Proposition 2.10. Consider $\phi \in \mathfrak{m}$ with associated tree potential $g_{\phi}$. Then

$$
\mathcal{J}\left(h+g_{\phi}\right)=\phi \mathcal{J}(h)
$$

for any tree potential $h \in \mathcal{P}$. 
2.2. Proofs. We now turn to the proofs. The fact that multiplier ideals are integrally closed is easy to establish.

Proof of Proposition 2.5. Let $\left(\psi_{i}\right)_{1}^{k}$ be generators of $I=\mathcal{J}(h)$. If $\psi \in \bar{I}$, then $\nu(\psi) \geq \min _{i}\left(\psi_{i}\right)$ for every $\nu \in \mathcal{V}_{\mathrm{qm}}$. This implies that

$$
\sup _{\nu} \frac{h(\nu)}{A(\nu)+\nu(\psi)} \leq \max _{i} \sup _{\nu} \frac{h(\nu)}{\nu\left(\psi_{i}\right)+A(\nu)}<1
$$

so that $\psi \in I$.

We now turn to the characterization of $\mathcal{J}(h)$ given in Proposition 2.4 The main point is that the supremum in the definition of $\mathcal{J}(h)$ is actually attained. In fact, the following lemma is the key to the openness conjecture for psh functions.

Lemma 2.11. Fix a tree potential $h \in \mathcal{P}$ and let $\psi \in R$. The function

$$
\chi(\nu)=\chi_{h, \psi}(\nu)=\frac{h(\nu)}{\nu(\psi)+A(\nu)},
$$

defined on $\mathcal{V}_{\mathrm{qm}}$, extends to a function on $\mathcal{V}$ continuous on segments. Its supremum is attained at a valuation $\nu_{\star}$. We may choose $\nu_{\star}$ to be a quasimonomial or a curve valuation, and to belong to the support of $h$. Further, we may take $\nu_{\star}$ such that:

(i) if $\psi \notin \mathfrak{m}$, then $\nu_{\star}$ is of multiplicity one;

(ii) if $h=g_{I}$ for some ideal $I$ in $R$, then $\nu_{\star}$ is an element in the approximating sequence of some valuation in $\operatorname{supp} \rho_{I}$; in particular, $\nu_{\star}$ is either divisorial or a curve valuation at an element of $R$;

(iii) if $h=g_{u}$ for some psh function $u$, then $\nu_{\star}$ is either quasimonomial or an analytic curve valuation.

The main observation in the proof of Lemma 2.11 is that it suffices to consider $h$ on a finite subtree of $\mathcal{V}$. In the same spirit we have:

Lemma 2.12. For $h \in \mathcal{P}$ and $0<\varepsilon<1$, the set

$$
\mathcal{T}=\mathcal{T}_{h, \varepsilon}:=\left\{\nu \in \mathcal{V}: \rho_{h}\{\mu \geq \nu\} \geq(1-\varepsilon) m(\nu)\right\}
$$

is a finite subtree of $\mathcal{V}$ and $\mathcal{J}(h)=\mathcal{J}\left(h_{\mathcal{T}}\right)$, where $h_{\mathcal{T}}$ is the smallest tree potential coinciding with $h$ on $\mathcal{T}$.

Proof of Lemma 2.11. We may assume that $\rho:=\Delta h$ has mass 1. Set

$$
\mathcal{T}=\mathcal{T}_{h, \psi}:=\left\{\nu: \rho\{\mu \geq \nu\} \geq \chi\left(\nu_{\mathfrak{m}}\right) m(\nu)\right\} .
$$

If $\nu \leq \nu^{\prime}$, then $m(\nu) \leq m\left(\nu^{\prime}\right)$ and $\rho\{\mu \geq \nu\} \geq \rho\left\{\mu \geq \nu^{\prime}\right\}$. Hence $\mathcal{T}$ is a tree. The number of ends of $\mathcal{T}$ is finite, bounded by $1 / \chi\left(\nu_{\mathfrak{m}}\right)$. Thus $\mathcal{T}$ is a finite tree. Its ends have finite multiplicity, hence consist of quasimonomial or curve valuations.

We claim that $\sup _{\mathcal{V}} \chi=\sup _{\mathcal{T}} \chi$. To see this, pick any $\nu_{1} \notin \mathcal{T}$. Let $\nu_{0}=$ $\max \left\{\nu \in \mathcal{T} ; \nu \leq \nu_{1}\right\}$ and let $\vec{v}$ be the tangent vector at $\nu_{0}$ represented by $\nu_{1}$. Write $m(\vec{v})=m, \alpha\left(\nu_{0}\right)=\alpha_{0}$ and, for $\nu \in\left[\nu_{0}, \nu_{1}\right], \alpha=\alpha(\nu)$. The right derivative of $h$ at $\nu_{0}$ with respect to the skewness equals $\rho U(\vec{v})$ (see Section 1.2.10). As $h$ is concave on $\left[\nu_{0}, \nu_{1}\right]$ we have $h(\nu) \leq h\left(\nu_{0}\right)+\rho U(\vec{v})\left(\alpha-\alpha_{0}\right)$ for $\nu \in\left[\nu_{0}, \nu_{1}\right]$. On the other hand, $A(\nu) \geq A\left(\nu_{0}\right)+m\left(\alpha-\alpha_{0}\right)$ and $\nu(\psi) \geq \nu_{0}(\psi)$; hence

$$
\chi(\nu) \leq \frac{h\left(\nu_{0}\right)+\rho U(\vec{v})\left(\alpha-\alpha_{0}\right)}{\nu_{0}(\psi)+A\left(\nu_{0}\right)+m\left(\alpha-\alpha_{0}\right)}:=M(\alpha), \quad \text { for all } \nu \in\left[\nu_{0}, \nu_{1}\right] .
$$


Here $\alpha \mapsto M(\alpha)$ is Möbius in $\alpha$; hence

$$
\sup _{\left[\nu_{0}, \nu_{1}\right]} \chi \leq \sup _{\left[\alpha_{0}, \infty\right]} M=\max \left\{\chi\left(\nu_{0}\right), m^{-1} \rho U(\vec{v})\right\} \leq \max \left\{\chi\left(\nu_{0}\right), \chi\left(\nu_{\mathfrak{m}}\right)\right\} .
$$

To prove the last inequality, we note that $U(\vec{v}) \cap \mathcal{T}=\emptyset$. If $\rho U(\vec{v})>m \chi\left(\nu_{\mathfrak{m}}\right)$, we could find $\nu^{\prime} \in U(\vec{v})$ close enough to $\nu_{0}$, of multiplicity $m$ and such that $\rho\{\mu \geq$ $\left.\nu^{\prime}\right\} \geq m \chi\left(\nu_{\mathfrak{m}}\right)$. This would imply $\nu^{\prime} \in \mathcal{T}$, a contradiction.

Thus $\sup _{\mathcal{V}} \chi=\sup _{\mathcal{T}} \chi$.

As each of the functions $h(\nu), \nu(\psi)$ and $A(\nu)$ is continuous on segments in $\mathcal{V}_{\mathrm{qm}}$, so is $\chi$. We extend $\chi$ to $\mathcal{V}$ by taking limits along segments. To see that these limits exist, pick $\nu_{0} \notin \mathcal{V}_{\mathrm{qm}}$ and let $\nu \rightarrow \nu_{0}$ along the segment $\left[\nu_{\mathfrak{m}}, \nu_{0}\right.$. If $\nu_{0}$ is a curve valuation, then $h(\nu) / \alpha(\nu) \rightarrow \rho\left\{\nu_{0}\right\}, \nu(\psi) / \alpha(\nu) \rightarrow \rho_{\psi}\left\{\nu_{0}\right\}$ and $A(\nu) / \alpha(\nu) \rightarrow m\left(\nu_{0}\right)$. This gives $\chi(\nu) \rightarrow \rho\left\{\nu_{0}\right\}\left(\rho_{\psi}\left\{\nu_{0}\right\}+m\left(\nu_{0}\right)\right)^{-1}$.

When $\nu_{0}$ is infinitely singular, we claim that $\chi$ is decreasing when $\nu \in\left[\nu_{\mathfrak{m}}, \nu_{0}\right]$ is sufficiently close to $\nu_{0}$. This implies that $\chi(\nu)$ converges when $\nu \rightarrow \nu_{0}$. To prove the claim, note that $\nu \mapsto \nu(\psi)$ is constant equal to $\nu_{0}(\psi)$ near $\nu_{0}$ and that the left derivative of $h$ with respect to skewness at $\nu$ equals $\rho\{\mu \geq \nu\}$. The left derivative of $\chi$ at $\nu$ is hence equal to

$$
\frac{d \chi}{d \alpha}=\frac{\left(A(\nu)+\nu_{0}(\psi)\right) \rho\{\mu \geq \nu\}-m(\nu) h(\nu)}{\left(A(\nu)+\nu_{0}(\psi)\right)^{2}} .
$$

By Section 1.2.8 $A(\nu)-m(\nu) \alpha(\nu) \rightarrow-\infty$ as $\nu \rightarrow \nu_{0}$ and $h(\nu) \geq \rho\{\mu \geq \nu\} \alpha(\nu)$. This easily implies $d \chi / d \alpha<0$ and completes the proof of the claim.

Thus the function $\chi$ extends to $\mathcal{V}$. Its restriction to the finite tree $\mathcal{T}$ is continuous; hence $\sup _{\mathcal{V}} \chi$ is attained at some $\nu_{\star} \in \mathcal{T}$.

Now suppose $\psi \notin \mathfrak{m}$, which amounts to $\nu(\psi) \equiv 0$. Consider a segment $] \nu_{0}, \nu_{1}[$ on which the multiplicity is constant equal to $m_{0} \geq 2$. Then (2.4) and the two inequalities $A(\nu)<m(\nu) \alpha(\nu)=m_{0} \alpha(\nu)$ and $h(\nu) \geq \rho\{\mu \geq \nu\} \alpha(\nu)$ imply $\frac{d \chi}{d \alpha}<0$ on $] \nu_{0}, \nu_{1}[$. This proves that $\chi$ is decreasing off the subtree $\{\nu: m(\nu)=1\}$. Hence $m\left(\nu_{\star}\right)=1$, proving (i).

We saw above that if $\nu$ is a curve valuation, then $\chi(\nu)=0$ unless $\rho$ puts mass on $\nu$. This proves (iii) as a measure represented by a psh function cannot charge formal curve valuations (see Section 1.4.5).

Finally, if $h=g_{I}$ for an ideal $I$, then $\rho$ is atomic, supported on finitely many valuations that are either divisorial or curve valuations; see Section 1.3. On any open segment in $\mathcal{V}$ not intersecting the support of $\rho$, and on which the multiplicity is constant, $h$ is Möbius, hence attains its maximum at its boundary points. This implies (ii).

Proof of Lemma 2.12. Suppose first that $\mathcal{T}$ is empty. This happens exactly when $h\left(\nu_{\mathfrak{m}}\right)<1-\varepsilon$. We have $A(\nu) \geq 1+\alpha(\nu)$ and $h(\nu) \leq h\left(\nu_{\mathfrak{m}}\right) \alpha(\nu)$ for all $\nu$, whence $\sup _{\mathcal{V}} \chi_{h, 1} \leq h\left(\nu_{\mathfrak{m}}\right)<1$ so that $\mathcal{J}(h)=\mathcal{J}\left(h_{\mathcal{T}}\right)=R$.

Suppose now that $\mathcal{T}$ is nonempty; i.e., $h\left(\nu_{\mathfrak{m}}\right) \geq 1-\varepsilon$. We follow the proof of Lemma 2.11 Clearly $\mathcal{T}$ is a subtree of $\mathcal{V}$ with at most $h\left(\nu_{\mathfrak{m}}\right) /(1-\varepsilon)$ ends. As $h \geq h_{\mathcal{T}}$ we have $\mathcal{J}(h) \subset \mathcal{J}\left(h_{\mathcal{T}}\right)$. To prove the reverse inclusion, we proceed as follows. Suppose $\sup _{\mathcal{T}} \chi=t<1$ where $\chi=\chi_{h, \psi}$. We must show that $\sup _{\mathcal{V}} \chi<1$. Consider $\nu_{0} \in \mathcal{T}$ and a segment $\left.] \nu_{0}, \nu_{1}\right]$, disjoint from $\mathcal{T}$. Let $m=m(\vec{v})$, where $\vec{v}$ is the tangent vector at $\nu_{0}$ represented by $\nu_{1}$. For $\left.\left.\nu \in\right] \nu_{0}, \nu_{1}\right]$ we get

$$
\chi(\nu) \leq \max \left\{\chi\left(\nu_{0}\right), \rho\{\mu \geq \nu\} / m\right\} \leq \max \{t, 1-\varepsilon\} .
$$


Hence $\sup _{\mathcal{V}} \chi \leq \max \{t, 1-\varepsilon\}<1$, which completes the proof.

Proof of Proposition 2.4. This is an immediate consequence of Lemma2.11 and the formula $\chi(\nu)=\rho_{h}\{\nu\} /\left(\rho_{\psi}\{\nu\}+m(\nu)\right)$ for a curve valuation $\nu$.

Next we address semicontinuity. The main ingredient in the proof is the following lemma. It can be viewed as a general statement about parameterized trees, but we shall formulate it only for the valuative tree $\mathcal{V}$ parameterized by skewness.

Lemma 2.13. Under the assumptions of Proposition 2.6, the functions $\frac{h_{n}(\nu)}{\alpha(\nu)} d e-$ crease uniformly on $\mathcal{V}_{\mathrm{qm}}$ to the function $h(\nu) / a(\nu)$.

Proof of Proposition 2.6. Clearly $\mathcal{J}\left(h_{n}\right)$ forms an increasing sequence of ideals in $R$ and $\mathcal{J}\left(h_{n}\right) \subset \mathcal{J}(h)$ for all $n$. Since $R$ is Noetherian, it suffices to show that $\bigcup \mathcal{J}\left(h_{n}\right) \supset \mathcal{J}(h)$. Consider $\phi \in \mathcal{J}(h)$ and write $\chi(\nu)=h(\nu) /(\nu(\phi)+A(\nu))$ and $\chi_{n}(\nu)=h_{n}(\nu) /(\nu(\phi)+A(\nu))$. Then $\sup \chi<1$. Since $A \geq \alpha$, Lemma 2.13 gives

$$
0 \leq \chi_{n}(\nu)-\chi(\nu) \leq \frac{h_{n}(\nu)}{\alpha(\nu)}-\frac{h(\nu)}{\alpha(\nu)} \rightarrow 0
$$

uniformly on $\mathcal{V}_{\mathrm{qm}}$, as $n \rightarrow \infty$. This implies $\sup \chi_{n}<1$, i.e., $\phi \in \mathcal{J}\left(h_{n}\right)$ for large $n$, completing the proof.

Proof of Lemma 2.13. As mentioned above, a version of the lemma holds for arbitrary parameterized trees. For a totally ordered tree it translates into the following elementary statement, the proof of which is left to the reader.

Lemma 2.14. Let $g_{n}, g$ be positive concave functions on $[1, \infty)$ such that $g_{n}$ decreases to $g$ pointwise. Then $g_{n}(x) / x$ decreases to $g(x) / x$ uniformly.

Of course, a tree such as $\mathcal{V}_{\mathrm{qm}}$ has a lot of branching. The following lemma will be used to control the behavior of tree potentials at branch points.

Lemma 2.15. Consider tree potentials $g, h$ and quasimonomial valuations $\mu, \nu$ with $\mu<\nu$. Assume that

$$
\frac{g(\nu)}{\alpha(\nu)} \geq \frac{h(\nu)}{\alpha(\nu)}+\varepsilon \quad \text { and } \quad \frac{g(\mu)}{\alpha(\mu)} \leq \frac{h(\mu)}{\alpha(\mu)}+\varepsilon
$$

for some $\varepsilon>0$. Then $\rho U(\vec{v}) \geq \varepsilon$, where $\rho=\rho_{g}$ is the tree measure of $g$ and $\vec{v}$ is the tangent vector at $\mu$ represented by $\nu$.

Proof. On the one hand, since $h$ is increasing, the assumptions give

$$
g(\nu) \geq h(\nu)+\varepsilon \alpha(\nu) \geq h(\mu)+\varepsilon \alpha(\nu)=h(\mu)+\varepsilon \alpha(\mu)+\varepsilon(\alpha(\nu)-\alpha(\mu)) .
$$

On the other hand, as $g$ is a tree potential, we have

$$
g(\nu) \leq g(\mu)+\rho U(\vec{v})(\alpha(\nu)-\alpha(\mu)) \leq h(\mu)+\varepsilon \alpha(\mu)+\rho U(\vec{v})(\alpha(\nu)-\alpha(\mu)) .
$$

Thus $\rho U(\vec{v}) \geq \varepsilon$.

We continue the proof of Lemma 2.13 and argue by contradiction. If $h_{n} / \alpha$ does not converge uniformly to $h / \alpha$, then, after passing to a subsequence if necessary, there exist $\nu_{n} \in \mathcal{V}_{\mathrm{qm}}$ and $\varepsilon>0$ such that $h_{n}\left(\nu_{n}\right) / \alpha\left(\nu_{n}\right)>h\left(\nu_{n}\right) / \alpha\left(\nu_{n}\right)+\varepsilon$ for all $n$. Using the compactness of $\mathcal{V}$ we may assume that $\nu_{n}$ converges weakly to some valuation $\nu_{\star} \in \mathcal{V}$. Since $h_{n}$ converges to $h$ pointwise on $\mathcal{V}_{\mathrm{qm}}$ we may assume that the $\nu_{n}$ are all distinct and different from $\nu_{\star}$. 
By Lemma 2.14 we may assume $h_{n} / \alpha \leq h / \alpha+\varepsilon$ on $\left[\nu_{\mathfrak{m}}, \nu_{\star}\left[\right.\right.$. Hence $\nu_{n} \notin\left[\nu_{\mathfrak{m}}, \nu_{\star}\right]$ so that $\mu_{n}:=\nu_{\star} \wedge \nu_{n}$ is quasimonomial and $\mu_{n}<\nu_{n}$. We apply Lemma 2.15 to $g=h_{n}, h=h, \mu=\mu_{k}$ and $\nu=\nu_{k}$, for $n \geq k$. Recall that $h_{n} \geq h_{k}$. We conclude that $\rho_{n} U_{k} \geq \varepsilon$, where $\rho_{n}=\rho_{h_{n}}$ and $U_{k}=U\left(\vec{v}_{k}\right)$, where $\vec{v}_{k}$ is the tangent vector at $\mu_{k}$ represented by $\nu_{k}$.

If $k \neq l$, then $U_{k}=U_{l}$ or $U_{k} \cap U_{l}=\emptyset$. As mass $\rho_{n}=h_{n}\left(\nu_{\mathfrak{m}}\right) \rightarrow h\left(\nu_{\mathfrak{m}}\right)$ is uniformly bounded, this implies that, after passing to a subsequence, all the $U_{k}$ are equal. In particular all the $\mu_{k}$ are equal. As $\mu_{k} \rightarrow \nu_{\star}$, this gives $\mu_{k}=\nu_{\star}$ for all $k$. In particular, $\nu_{\star}$ is quasimonomial and $\nu_{n}>\nu_{\star}$.

For $k>1$ we have $\left.\left.\nu_{k} \wedge \nu_{1} \in\right] \nu_{\star}, \nu_{1}\right]$ as $U_{k}=U_{1}$. Again by Lemma 2.14 $\nu_{k} \wedge \nu_{1}<\nu_{k}$ for all $k \geq 2$. As $\nu_{1} \wedge \nu_{k} \rightarrow \nu_{\star}$ as $k \rightarrow \infty$ we may assume that the valuations $\nu_{1} \wedge \nu_{k}$ are all distinct. We again apply Lemma 2.15, now with $\mu=\nu_{1} \wedge \nu_{k}, \nu=\nu_{k}$ and still using $h=h$ and $g=h_{n}$ for $n \geq k$. We conclude that $\rho_{n} U_{k}^{\prime} \geq \varepsilon$, where $U_{k}^{\prime}=U\left(\vec{v}_{k}^{\prime}\right)$ and $\vec{v}_{k}^{\prime}$ is the tangent vector at $\nu_{1} \wedge \nu_{k}$ represented by $\nu_{k}$. But the regions $U_{k}^{\prime}$ are mutually disjoint, so since the $\rho_{n}$ has uniformly bounded mass, this gives a contradiction.

Next we prove the bounds for the singularity exponents.

Proof of Proposition 2.7. Recall that $\rho$ has mass $h\left(\nu_{\mathfrak{m}}\right)$.

Write $\chi(\nu)=h(\nu) / A(\nu)$ so that $\lambda(h)=\sup \chi$. The lower bound $\lambda(h) \geq \chi\left(\nu_{\mathfrak{m}}\right)=$ $\frac{1}{2} h\left(\nu_{\mathfrak{m}}\right)$ is immediate. Recall that $A(\nu) \geq 1+\alpha(\nu)$. If $\nu \in \mathcal{V}_{\mathrm{qm}}, \nu \neq \nu_{\mathfrak{m}}$, then $h(\nu) \leq h\left(\nu_{\mathfrak{m}}\right)+\rho U(\vec{v})(\alpha(\nu)-1)$, where $\vec{v}$ is the tangent vector at $\nu_{\mathfrak{m}}$ represented by $\nu$. In general, this gives $h(\nu) \leq h\left(\nu_{\mathfrak{m}}\right) \alpha(\nu)$, so that $\chi(\nu)<h\left(\nu_{\mathfrak{m}}\right)$.

If $\rho U(\vec{v}) \leq \frac{1}{2} h\left(\nu_{\mathfrak{m}}\right)$, then $h(\nu) \leq \frac{1}{2} h\left(\nu_{\mathfrak{m}}\right)(1+\alpha(\nu))$ so that $\chi(\nu) \leq \frac{1}{2} h\left(\nu_{\mathfrak{m}}\right)$. On the other hand, if $\rho U(\vec{v})>\frac{1}{2} h\left(\nu_{\mathfrak{m}}\right)$, then we may move $\nu$ closer to $\nu_{\mathfrak{m}}$, still keeping $\nu \in U(\vec{v})$, so that $m(\nu)=1, A(\nu)=1+\alpha(\nu)$ and $h(\nu)>h\left(\nu_{\mathfrak{m}}\right)+\frac{1}{2} h\left(\nu_{\mathfrak{m}}\right)(\alpha(\nu)-1)$. This gives $\lambda(h) \geq \chi(\nu)>\frac{1}{2} h\left(\nu_{\mathfrak{m}}\right)$.

Finally suppose $\lambda(h)=h\left(\nu_{\mathfrak{m}}\right)$. By Lemma 2.11 we have $\lambda(h)=\chi(\nu)$ for some valuation $\nu \in \mathcal{V}$ of multiplicity one. We have seen above that $\chi<h\left(\nu_{\mathfrak{m}}\right)$ on $\mathcal{V}_{\mathrm{qm}}$. Hence $\nu$ is a curve valuation. But then $\chi(\nu)=\rho\{\nu\} / m(\nu)=\rho\{\nu\}$ so that $\rho\{\nu\}=h\left(\nu_{\mathfrak{m}}\right)$ and $\rho$ is a point mass at $\nu$.

Finally we prove the second version of Skoda's Theorem.

Proof of Proposition 2.10. By unique factorization we may assume that $\phi$ is irreducible. We first claim that $J\left(h+g_{\phi}\right) \subset \phi R$. Indeed, if $\psi \in \mathcal{J}\left(h+g_{\phi}\right)$, then by Proposition 2.4 we have $\rho_{\psi}\left\{\nu_{\phi}\right\}+m(\phi)>\rho_{h}\left\{\nu_{\phi}\right\}+\rho_{\phi}\left\{\nu_{\phi}\right\} \geq 0+m(\phi)$. Thus $\rho_{\psi}\left\{\nu_{\phi}\right\}>0$ so that $\phi$ divides $\psi$.

It hence suffices to show that if $\psi \in R$, then $\phi \psi \in \mathcal{J}\left(h+g_{\phi}\right)$ iff $\psi \in \mathcal{J}(h)$. But this follows easily from Proposition 2.4. Indeed, if $\nu$ is quasimonomial, then $\nu(\psi \phi)-\left(h+g_{\phi}\right)(\nu)=\nu(\psi)-h(\nu)$ and if $\nu$ is a curve valuation, then $\rho_{\psi \phi}\{\nu\}-$ $\left(\rho_{h}+\rho_{\phi}\right)\{\nu\}=\rho_{\psi}\{\nu\}-\rho_{h}\{\nu\}$.

\section{Multiplier ideals of ideAls}

We now show that if $h$ is the tree transform of a formal power $I^{c}$, where $I$ is an ideal and $c>0$, then the multiplier ideal of $h$ agrees with the multiplier ideal of $I^{c}$ with the standard definitions given in the literature. First we consider the approach based on resolution of singularities as described in [La Part III]. Then we explain how to recover Lipman's definition given in Li]. Finally we show how 
to understand asymptotic multiplier ideals of graded systems of ideals in terms of tree potentials.

3.1. Multiplier ideals through log-resolutions. The exposition follows La Part III]. See also [LW] and [TW].

A log-resolution of an ideal $I \subset R$ is a birational morphism $\pi: X \rightarrow \operatorname{Spec} R$ such that $X$ is non-singular and $I \cdot \mathcal{O}_{X}$ is locally principal: $I \cdot \mathcal{O}_{X}=\mathcal{O}_{X}(-F)$ for an effective divisor $F$, and $F+E$ has simple normal crossing singularities, where $E$ is the exceptional divisor of $\pi$.

The existence of such a resolution in our setting is well known. In fact, $\pi$ can be chosen as a composition of blowups at (closed) points. In the analytic case $R=\mathcal{O}_{0}$ the reader may think of Spec $R$ as a neighborhood of the origin in $\mathbf{C}^{2}$.

Another ingredient in the definition is the relative canonical divisor of $\pi$. It is the unique divisor $K_{X / R}$, with support in $E$ and whose class represents $J \pi$, the Jacobian determinant of $\pi$ :

$$
K_{X / R}=K_{X}-\pi^{*} K_{\text {Spec } R} .
$$

Here $K_{X}$ and $K_{\text {Spec } R}$ denote the sheaf of 2-differentials over $X$ and $\operatorname{Spec} R$, respectively.

We also denote by $\lfloor c\rfloor$ the round-down of a positive real number, i.e., the greatest integer less than or equal to $c$.

Definition 3.1. Let $I \subset R$ be an ideal, and $c$ be a positive real number. Fix a log-resolution $\pi: X \rightarrow$ Spec $R$ of $I$ such that $I \cdot \mathcal{O}_{X}=\mathcal{O}_{X}(-F)$ with $F=\sum r_{i} E_{i}$, and write $K_{X / R}=\sum \beta_{i} E_{i}$. We define the multiplier ideal of $I^{c}$ to be

$$
\mathcal{J}\left(I^{c}\right):=\left\{\psi \in R: \operatorname{div}_{E_{i}}\left(\pi^{*} \psi\right) \geq\left\lfloor c r_{i}\right\rfloor-\beta_{i} \text { for all } i\right\} .
$$

Here $\operatorname{div}_{E_{i}}$ denotes the generic order of vanishing along $E_{i}$ of a function on $X$.

This definition does not depend on the choice of log-resolution. In the analytic case $R=\mathcal{O}_{0}$, the definition says that a holomorphic germ $\psi$ belongs to the multiplier ideal iff the pull-back of $\psi$ vanishes to sufficiently high orders along the irreducible components of $\pi^{-1}(V)$, where $V=\bigcap_{\phi \in I} \phi^{-1}(0)$.

Proposition 3.2. For any ideal $I \subset R$ and any positive real number $c>0$, the multiplier ideal $\mathcal{J}\left(I^{c}\right)$ as defined in Definition 3.1 coincides with the multiplier ideal of the tree transform $c g_{I}$ as defined in Definition 2.1.

Remark 3.3. Similarly, we may associate a multiplier ideal to an expression of the form $I_{1}^{c_{1}} \cdots I_{n}^{c_{n}}$ for ideals $I_{j}$ and real numbers $c_{j}$. The proof of Proposition 3.2 is easily adapted to show that $J\left(I_{1}^{c_{1}} \cdots I_{n}^{c_{n}}\right)=J\left(\sum_{j} c_{j} g_{I_{j}}\right)$.

Proof. We first need to translate the condition of vanishing of $\pi^{*} \psi$ along $E_{i} \subset X$ in terms of valuations. We shall denote this condition by $(\star)_{i}$. Note that $\operatorname{div}_{E_{i}}$ is a valuation with values in $\mathbf{Z}$. We have $r_{i}=\operatorname{div}_{E_{i}}\left(\pi^{*} I\right)$ and $\beta_{i}=\operatorname{div}_{E_{i}}(J \pi)$ where $J \pi$ is the Jacobian determinant of $\pi$.

First suppose $E_{i}$ does not belong to the exceptional divisor of $\pi$. Its image by $\pi$ is then an irreducible curve. We let $\nu_{i} \in \mathcal{V}$ be the associated curve valuation (see Section 1.2.2) and $m_{i}$ its multiplicity. Denote by $\rho_{I}$ and $\rho_{\psi}$ the tree measures of $I$ and $\psi$, respectively. It follows from Section 1.3 that $\operatorname{div}_{E_{i}}\left(\pi^{*} I\right)$ is equal to $m_{i}^{-1} \rho_{I}\left\{\nu_{i}\right\}$. In the same way, $\operatorname{div}_{E_{i}}\left(\pi^{*} \psi\right)=m_{i}^{-1} \rho_{\psi}\left\{\nu_{i}\right\}$. On the other hand, it is clear that $\beta_{i}=\operatorname{div}_{E_{i}}(J \pi)=0$. The condition $(\mathbb{\nabla})_{i}$ is hence equivalent to 
$m_{i}^{-1} \rho_{\psi}\left\{\nu_{i}\right\} \geq\left\lfloor c m_{i}^{-1} \rho_{I}\left\{\nu_{i}\right\}\right\rfloor$. As the left-hand side is an integer, this amounts to $m_{i}^{-1} \rho_{\psi}\left\{\nu_{i}\right\}>c m_{i}^{-1} \rho_{I}\left\{\nu_{i}\right\}-1$. We thus conclude that if $E_{i} \not \subset \pi^{-1}(0)$, then

$$
(\star)_{i} \Leftrightarrow \rho_{\psi}\left\{\nu_{i}\right\}+m\left(\nu_{i}\right)>c \rho_{I}\left\{\nu_{i}\right\} .
$$

Now suppose $E_{i}$ is an irreducible component of $\pi^{-1}(0)$. Then $\pi_{*} \operatorname{div}_{E_{i}}(\psi):=$ $\operatorname{div}_{E_{i}}\left(\pi^{*} \psi\right)$ defines a divisorial valuation with values in $\mathbf{Z}$. It is proportional to a unique normalized valuation $\nu_{i} \in \mathcal{V}: \pi_{*} \operatorname{div}_{E_{i}}=b_{i} \nu_{i}$ where $b_{i}=b\left(\nu_{i}\right)$ is the generic multiplicity of $\nu_{i}$ (see Section 1.2.9). The thinness $A\left(\nu_{i}\right)$ is given by $a_{i} / b_{i}$, where $a_{i}=a\left(\nu_{i}\right)=\operatorname{div}_{E_{i}}(J \pi)+1$ (again by Section 1.2.9). We infer that if $E_{i} \subset \pi^{-1}(0)$, then

$$
\begin{aligned}
(\star)_{i} & \Leftrightarrow \operatorname{div}_{E_{i}}\left(\pi^{*} \psi\right) \geq\left\lfloor c \operatorname{div}_{E_{i}}\left(\pi^{*} I\right)\right\rfloor-\operatorname{div} E_{i}(J \pi) \\
& \Leftrightarrow \operatorname{div}_{E_{i}}\left(\pi^{*} \psi\right)>c \operatorname{div}_{E_{i}}\left(\pi^{*} I\right)-\operatorname{div} E_{i}(J \pi)-1 \\
& \Leftrightarrow b_{i} \nu_{i}(\psi)>c b_{i} \nu_{i}(I)-a_{i},
\end{aligned}
$$

i.e.,

$$
(\star)_{i} \Leftrightarrow \nu_{i}(\psi)+A\left(\nu_{i}\right)>c g_{I}\left(\nu_{i}\right) .
$$

Now consider $\psi \in \mathcal{J}\left(c g_{I}\right)$. It follows immediately from Proposition 3.2 that condition $(\star)_{i}$ in (3.1), (3.2) holds for all $i$. Thus $\psi \in \mathcal{J}\left(I^{c}\right)$.

Conversely suppose $\psi \notin \mathcal{J}\left(c g_{I}\right)$. Again we use Proposition 2.4. This tells us that either (a) or (b) fails in that proposition. If (b) fails, then there exists a curve valuation $\nu$ such that $\rho_{\psi}\{\nu\}+m(\nu) \leq c \rho_{I}\{\nu\}$. This inequality implies that the element in $R$ corresponding to $\nu$ is a factor of $I$. Hence $\nu$ is one of the curve valuations $\nu_{i}$ above. Thus $(\star)_{i}$ in (3.1) fails and $\psi \notin \mathcal{J}\left(I^{c}\right)$. On the other hand, if (a) fails, then there exists a divisorial valuation $\nu$ such that $\nu(\psi)+A(\nu) \leq c \nu(I)$. We may take a log-resolution $\pi$ of $I$ such that $\nu$ corresponds to an exceptional divisor of $\pi$. This can be achieved by further blowups of a given log-resolution of $I$. Thus $\nu=\nu_{i}$ for some $i$, so that $(\star)_{i}$ in (3.2) fails and $\psi \notin \mathcal{J}\left(I^{c}\right)$.

3.2. Lipman's approach. The definition of Lipman 6 in [Li] has the advantage of not depending on a log-resolution and hence makes sense in great generality.

We describe Lipman's construction in our setting, namely for an equicharacteristic zero, two-dimensional, regular local ring $R$ with algebraically closed residue field $k$. Write $K$ for the fraction field of $R$.

We first need some definitions. For a (not necessarily centered) valuation $\mu$ on $R$, we let $R_{\mu}=\{\phi \in K: \mu(\phi) \geq 0\}$ be the valuation ring, $\mathfrak{m}_{\mu}=\{\phi \in K: \mu(\phi)>0\}$ the (unique) maximal ideal in $R_{\mu}$ and $k_{\mu}:=R_{\mu} / \mathfrak{m}_{\mu}$ the residue field. Note that $k_{\mu}$ is a field extension of $k$. A prime divisor is by definition a valuation on $R$ whose residue field has transcendence degree $l-1$ over $k$, where $l$ is the height of $\mathfrak{m}_{\mu} \cap R$, i.e., the dimension of the center of $\mu$ in Spec $R$. Let us describe explicitly in our situation what a prime divisor means. Two different cases may appear.

(i) $l=1$. Then the center of $\mu$ is a principal ideal generated by an irreducible element $\psi \in R$. In this case for any $\phi \in R$, we have $\mu(\phi)=\operatorname{div}_{C}(\phi)$ for some irreducible $C \subset \operatorname{Spec} R$.

(ii) $l=2$. Then $\mu$ is centered at the maximal ideal $\mathfrak{m}$ of $R$. The transcendence degree of $k_{\mu}$ being 1 implies that $\mu$ is a divisorial valuation (see [FJ1. Proposition 1.12]).

${ }^{6}$ Lipman used the term adjoint ideals and defined them for ideals rather than formal powers of ideals. 
Lipman's construction uses the Jacobian ideal $J_{R_{\mu} / R}$, whose definition is purely algebraic. In our setting it is given as follows, following the two cases above:

(i) When $l=1$, the ideal $J_{R_{\mu} / R}$ is trivial.

(ii) When $l=2, \mu$ is a divisorial valuation. Fix a composition of blowups $\pi: X \rightarrow \operatorname{Spec} R$ such that the center of $\mu$ in $X$ is a divisor $E$. For a closed point $p \in E$, the ring $\mathcal{O}_{X, p}$ can be naturally viewed as a subring of $R_{\mu}$ by the isomorphism $\pi_{*}$ between function fields of $X$ and $\operatorname{Spec} R$. Then the ideal $J_{R_{\mu} / R} \subset R_{\mu}$ is generated by the Jacobian determinant of $\pi$.

We can now set

Definition 3.4. Let $I \subset R$ be an ideal and let $c>0$. The multiplier ideal of $I^{c}$ is the ideal

$$
\mathcal{J}\left(I^{c}\right):=\bigcap_{\mu}\left\{\psi \in R: \mu(\psi) \geq\lfloor c \mu(I)\rfloor-\mu\left(J_{R_{\mu} / R}\right)\right\}
$$

where the intersection is taken over all prime divisors $\mu$ of $R 7$

If $I$ is an ideal and $c>0$, then we may consider both the multiplier ideal of $I^{c}$ above and the multiplier ideal of the tree potential $c g_{I}$. Both are defined in terms of valuations. Hence the following result is perhaps not so surprising.

Proposition 3.5. For any ideal $I \subset R$ and any $c>0$, the multiplier ideal $\mathcal{J}\left(I^{c}\right)$ in the sense of Definition 3.4 coincides with the multiplier ideal of the tree potential $c g_{I}$ as defined in Definition 2.1 .

Proof. Pick $\psi \in R$. First consider a curve valuation $\nu=\nu_{C}$ in $\mathcal{V}$. We denote the prime divisor associated to $\nu$ by $\operatorname{div}_{C}$. Note that $\operatorname{div}_{C}$ is not centered at the maximal ideal $\mathfrak{m} \subset R$. We then have the sequence of equivalences

$$
\begin{aligned}
\operatorname{div}_{C}(\psi) \geq\left\lfloor c \operatorname{div}_{C}(I)\right\rfloor-\operatorname{div}_{C}\left(J_{R_{\operatorname{div}_{C}} / R}\right) & \Leftrightarrow \operatorname{div}_{C}(\psi)>c \operatorname{div}_{C}(I)-1 \\
& \Leftrightarrow \rho_{\psi}\left\{\nu_{C}\right\}>c \rho_{I}\left\{\nu_{C}\right\}-m(C) .
\end{aligned}
$$

Now consider a divisorial valuation $\nu \in \mathcal{V}$. Fix a composition of blowups $\pi$ such that $b \nu=\pi_{*} \operatorname{div}_{E}$ for some exceptional component $E, b$ being the generic multiplicity of $\nu$ (see Section 1.2.9). Let $a-1$ be the order of vanishing of the Jacobian determinant of $\pi$ along $E$. By the discussion above,

$$
\begin{aligned}
\nu(\psi) \geq\lfloor c \nu(I)\rfloor-\nu\left(J_{R_{\nu} / R}\right) & \Leftrightarrow \operatorname{div}_{E}\left(\pi^{*} \psi\right) \geq\left\lfloor c \operatorname{div}_{E}\left(\pi^{*} I\right)\right\rfloor-\operatorname{div}_{E}(J \pi) \\
& \Leftrightarrow \operatorname{div}_{E}\left(\pi^{*} \psi\right)>c \operatorname{div}_{E}\left(\pi^{*} I\right)-\operatorname{div}_{E}(J \pi)-1 \\
& \Leftrightarrow b \nu(\psi)>b c \nu(I)-a \\
& \Leftrightarrow \nu(\psi)>c g_{I}(\nu)-A(\nu) .
\end{aligned}
$$

This concludes the proof in view of Proposition 2.4

3.3. Multiplier ideals of graded systems. Another type of multiplier ideals has been defined for graded systems of ideals. Here we show that these multiplier ideals can also be understood through tree potentials.

Let $I_{\bullet}=\left(I_{k}\right)_{k \geq 1}$ be a graded system of ideals. This means that $I_{k}$ is an ideal in $R$ for every $k$ and that $I_{k} I_{l} \subset I_{k+l}$ for all $k, l$. Fix $c>0$ rational. It is proved in La (see also below) that the sequence of ideals $\left(\mathcal{J}\left(I_{k}^{c / k}\right)\right)_{k \geq 1}$ has a unique maximal element.

\footnotetext{
${ }^{7}$ Lipman's original definition was for $c=1$ in which case the " $\lfloor\cdot \mid$ " can be omitted.
} 
Definition 3.6. The asymptotic multiplier ideal $\mathcal{J}\left(I_{\bullet}^{c}\right)$ is the unique maximal element of the sequence $\left(\mathcal{J}\left(I_{k}^{c / k}\right)\right)_{k \geq 1}$.

Definition 3.7. The singularity exponent (or log-canonical threshold) of $I_{\bullet}$ is $c\left(I_{\bullet}\right):=\sup \left\{c>0: \mathcal{J}\left(I_{\bullet}^{c}\right)=R\right\}$. The Arnold multiplicity is $\lambda\left(I_{\bullet}\right):=c\left(I_{\bullet}\right)^{-1}$.

Let $g_{k}$ be the tree transform of $I_{k}$. The condition $I_{k} I_{l} \subset I_{k+l}$ then implies $g_{k+l} \leq g_{k}+g_{l}$. It is then elementary that the sequence $k^{-1} g_{k}$ converges in $\mathcal{P}$ (i.e., pointwise) to the tree potential $g:=\inf k^{-1} g_{k}$. Moreover, the subsequence $2^{-j} g_{2^{j}}$ is decreasing, so by semicontinuity (Proposition 2.6) we have $\mathcal{J}\left(c 2^{-j} g_{2^{j}}\right)=\mathcal{J}(c h)$ for $j \gg 0$. On the other hand, $\mathcal{J}\left(c k^{-1} g_{k}\right)=\mathcal{J}\left(I_{k}^{c / k}\right)$ for all $k$ by Proposition 3.2 This gives

Proposition 3.8. Given a graded sequence $I_{\bullet}$ of ideals there exists a tree potential $g=g_{I_{\bullet}}$ such that the asymptotic multiplier ideal $\mathcal{J}\left(I_{\bullet}^{c}\right)$ coincides with the multiplier ideal $\mathcal{J}(c g)$ for any $c>0$. As a consequence, $c\left(I_{\bullet}\right)=c(g)$ and $\lambda\left(I_{\bullet}\right)=\lambda(g)$.

Remark 3.9. It follows from Theorem 8.1 that $g=g_{I}$. is uniquely determined by the properties stated in Proposition 3.8

Remark 3.10. Not every tree potential $g$ is associated to a graded system of ideals I. An example is given by $g=\alpha(\mu \wedge \cdot)$, where $\mu$ is infinitely singular of infinite skewness. Indeed, $g(\mu)=\infty$, whereas $\mu(I)<\infty$ for every ideal $I$; hence $g_{I_{\bullet}}(\mu)<\infty$ for any graded system $I_{\bullet}$. It would be interesting to characterize all tree potentials associated to graded systems of ideals. See [FJ2, Section 6.2] for related questions.

Example 3.11. If $I \subset R$ is a fixed ideal and $k_{0} \in \mathbf{N}$, then $I_{k}:=I^{k+k_{0}}$ defines a graded system of ideals. In this case $k^{-1} g_{I_{k}}=\left(1+k_{0} / k\right) g_{I} \rightarrow g_{I}$ so $\mathcal{J}\left(I_{\bullet}^{c}\right)=\mathcal{J}\left(I^{c}\right)$ independently of $k_{0}$.

Example 3.12. If $\nu \in \mathcal{V}$, then $I_{k}:=\{\phi \in R: \nu(\phi) \geq k\}$ defines a graded system of ideals. In this case the tree potential $h$ in Proposition 3.8 satisfies

$$
\Delta h=\alpha(\nu)^{-1} \nu .
$$

Moreover, $c\left(I_{\bullet}\right)=\alpha(\nu)\left(1+\alpha\left(\nu_{1}\right)^{-1}\right)$, where $\nu_{1}$ is the first element in the approximating sequence of $\nu$. In particular, if $\alpha(\nu)=\infty$, then $g=0$, and $c\left(I_{\bullet}\right)=\infty$.

Let us sketch a proof of (3.3). It is easy to see in general that $g(\mu) \geq h(\mu):=$ $\alpha(\nu)^{-1} \alpha(\mu \wedge \nu)$. For the reverse inequality, suppose first that $\nu$ is a divisorial valuation of generic multiplicity $b$. Then the tree transform of $I_{b \alpha(\nu)}$ is precisely $\mu \rightarrow b \alpha(\mu \wedge \nu)$; see Section 1.3. Whence $g=h$ in this case. In general, take an increasing sequence of divisorial valuations $\nu_{n}$ tending to $\nu$, and apply the preceding result.

\section{Multiplier IDEALS OF PSH FUnCTIONS}

We now turn to multiplier ideals of psh functions. For this to make sense we work in the analytic case $R=\mathcal{O}_{0}$. Recall that if $u$ is psh, then the multiplier ideal $\mathcal{J}(u)$ consists of the holomorphic germs $\psi \in R$ such that $|\psi|^{2} e^{-2 u}$ is locally integrable at the origin (see $[\mathrm{N},[\mathrm{DK}]$ ). The (complex) singularity exponent (or log-canonical threshold) of $u$ at the origin is $c(u)=\sup \left\{c>0: e^{-2 c u} \in L_{\text {loc }}^{1}\right\}$. The Arnold multiplicity is $\lambda(u)=c(u)^{-1}$. Our goal is to prove the following theorem. 
Theorem 4.1. If $u$ is a psh function, then the multiplier ideal of $u$ equals that of its tree transform. In other words, if $\psi$ is a holomorphic germ, then

$$
\psi \in \mathcal{J}(u) \quad \text { iff } \sup _{\nu \in \mathcal{V}_{\mathrm{qm}}} \frac{\nu(u)}{\nu(\psi)+A(\nu)}<1 .
$$

The proof makes essential use of the Demailly approximation technique in Section 1.4.4 In fact we have

Corollary 4.2. If $u_{n}$ are the Demailly approximants of $u$, then $\mathcal{J}\left(u_{n}\right)=\mathcal{J}(u)$ for $n \gg 1$.

Proof. We have $0 \leq \nu(u)-\nu\left(u_{n}\right) \leq A(\nu) / n$ for all $\nu \in \mathcal{V}_{\mathrm{qm}}$; hence

$$
0 \leq \frac{\nu(u)}{\nu(\psi)+A(\nu)}-\frac{\nu\left(u_{n}\right)}{\nu(\psi)+A(\nu)} \leq \frac{1}{n},
$$

which easily implies the corollary in view of (4.1).

We split the proof of the theorem into two parts: non-integrability and integrability. Set

$$
\chi(\nu)=\chi_{u, \psi}(\nu)=\frac{\nu(u)}{\nu(\psi)+A(\nu)} .
$$

By Lemma 2.11, $\chi$ attains it supremum on $\mathcal{V}$. We emphasize that this fact is really the key to the openness conjecture (Corollary 5.1). At any rate, Theorem 4.1 follows immediately from Proposition 4.3 and Proposition 4.4 below.

Proposition 4.3. Let $u$ and $\psi$ be as in Theorem 4.1. Suppose there exists $\nu \in \mathcal{V}$ such that $\chi(\nu) \geq 1$. Then $|\psi|^{2} e^{-2 u}$ is not locally integrable at the origin. In fact, we then have $\operatorname{Vol}\left\{|\psi|^{2} e^{-2 u}>R\right\} \gtrsim R^{-1}$ as $R \rightarrow \infty$.

Proposition 4.4. Let $u$ and $\psi$ be as in Theorem 4.1 Suppose that $\sup \chi<1$. Then $|\psi|^{2} e^{-2 u}$ is locally integrable at the origin.

Proof of Proposition 4.3. It suffices to show the estimate $\operatorname{Vol}\left\{|\psi|^{2} e^{-2 u}>R\right\} \gtrsim R^{-1}$ as $R \rightarrow \infty$. By Lemma 2.11, we may assume $\nu$ is not infinitely singular.

First suppose $\nu$ is quasimonomial. Then $\nu(\psi)-\nu(u) \leq-A(\nu)<0$. We use the analysis in Section 1.4.2, Write $\nu=\nu_{\phi, t}$ and pick a coordinate $x$ transverse to $\phi$. Let $\mathcal{A}=\mathcal{A}_{\phi, t, x, C}(r)$ be a characteristic region. Then $\operatorname{Vol}(\mathcal{A}) \gtrsim r^{2 A(\nu)}$. Moreover, $u(p) \leq \nu(u) \log \|p\| r+O(1)$ and $\log |\psi| \geq \nu(\psi) \log \|p\|+O(1)$, so

$$
\log |\psi|-u \geq(\nu(\psi)-\nu(u)) \log \|p\|+O(1) \geq-A(\nu) \log r+O(1)
$$

in $\mathcal{A}$. The desired estimate follows by choosing $R \sim r^{-2 A(\nu)}$.

Now suppose $\nu=\nu_{\phi}$ is a curve valuation. Then $\phi$ is a holomorphic germ. By Section 1.4.5. we have $u=c \log |\phi|+u^{\prime}$ with $c=\rho_{u}\left\{\nu_{\phi}\right\} / m(\phi)$ and $u^{\prime}$ psh. Similarly, $\psi=\phi^{a} \psi^{\prime}$, where $a \geq 0$ and $\phi$ does not divide $\psi^{\prime}$. Then $1 \leq \chi\left(\nu_{\phi}\right)=c /(a+1)$, so that $c \geq a+1$. Since $u^{\prime}$ is bounded from above we have

$$
|\psi|^{2} e^{-2 u} \gtrsim|\phi|^{2(a-c)}\left|\psi^{\prime}\right|^{2} \gtrsim \frac{\left|\psi^{\prime}\right|^{2}}{|\phi|^{2}}
$$

near the origin. Pick a composition of blowups $\pi$, and a smooth point $p \in \pi^{-1}(0)$ such that the strict transform of $\phi^{-1}(0)$ is smooth and intersects the exceptional divisor transversely at $p$, and such that the strict transform of $\left(\psi^{\prime}\right)^{-1}(0)$ does not contain $p$. This can be done by successively blowing up the intersection point of the 
strict transform of $\phi^{-1}(0)$ and the exceptional divisor. Fix coordinates $(z, w)$ at $p$ such that $\pi^{-1}(0)=\{z=0\}$ and $\phi^{-1}(0)=\{z w=0\}$. We may assume $\pi^{*} \phi=z^{k} w$, $J \pi=z^{l}$ and $\pi^{*} \psi^{\prime}=z^{m} \tilde{\psi}$, where $k, l, m \in \mathbf{N}^{*}$ and $\tilde{\psi}(0) \neq 0$. Fix $r_{0}>0$ small, and consider $0<r \ll r_{0}$. Set $\Omega(r):=\pi\left\{|z|<r_{0},|w|<r\right\}$. By the change of variables formula, $\operatorname{Vol} \Omega(r) \gtrsim r^{2}$. If $p=\pi(z, w) \in \Omega(r)$, then

$$
|\psi(p)|^{2} e^{-2 u(p)} \gtrsim \frac{\left|\pi^{*} \psi^{\prime}(z, w)\right|^{2}}{\left|\pi^{*} \phi(z, w)\right|^{2}} \gtrsim|w|^{-2} \geq r^{-2} .
$$

The desired estimate follows by choosing $R \sim r^{-2}$.

Proof of Proposition 4.4. First suppose $u$ has logarithmic singularities, say $u=$ $\frac{c}{2} \log \sum_{l=1}^{k}\left|\phi_{l}\right|^{2}$ for $\phi_{i} \in R$ and $c>0$. The tree transform of $u$ then coincides with the tree transform of $I^{c}$, where $I$ is the ideal generated by the $\phi_{i}$ 's. Integrability can now be proved as in [DK] Proposition 1.7], using the results of Section 3 ,

This goes as follows. Let $\pi: X \rightarrow\left(\mathbf{C}^{2}, 0\right)$ be a log-resolution of the ideal $I$. Thus the total transform of the curve $V=\left\{\prod_{l} \phi_{l}=0\right\}$ is a union of smooth components with normal crossing singularities. By the change of variables formula, the function $\Phi^{2}=|\psi|^{2} \exp (-2 u)$ is locally integrable iff $\left(\pi^{*} \Phi \cdot|J \pi|\right)^{2}$ is locally integrable at any point $p \in \pi^{-1}(0)$.

Let $\left\{E_{i}\right\}$ be the set of irreducible components of $\pi^{-1}(V)$. For any $i$, we let $\beta_{i}$, $r_{i}$ and $\gamma_{i}$ be the order of vanishing along $E_{i}$ of $J \pi, \pi^{*} I$ and $\pi^{*} \psi$, respectively. As in Section 3 the condition $\sup \chi<1$ implies that $\delta_{i}:=\gamma_{i}+\beta_{i}-\left\lfloor c r_{i}\right\rfloor \geq 0$; hence $\delta_{i}>-1$ for all $i$.

Now pick $p \in \pi^{-1}(0)$ and local coordinates $(z, w)$ at $p$ such that $\pi^{-1} V \subset\{z w=$ $0\}$. The calculations above give $\pi^{*} \Phi \cdot|J \pi| \gtrsim|z|^{\delta}|w|^{\delta^{\prime}}$ where $\delta, \delta^{\prime}>-1$. Thus $\left(\pi^{*} \Phi \cdot|J \pi|\right)^{2}$ is locally integrable at $p$, completing the proof when $u$ has logarithmic singularities.

We now consider the case of an arbitrary psh function. As in the proof of Theorem 4.2 in [DK] we use Demailly approximation to reduce to the preceding case. By Section 1.4.4there exists a small neighborhood $B^{\prime}$ of the origin, and, for each $n>$ 0 , finitely many holomorphic functions $\left\{g_{n l}\right\}_{l=1}^{k}$ with $\int_{B^{\prime}}\left|g_{n l}\right|^{2} \exp (-2 n u) \leq 1$, such that the psh function $u_{n}=(2 n)^{-1} \log \sum\left|g_{n l}\right|^{2}$ (which has logarithmic singularities) satisfies $\left|\nu(u)-\nu\left(u_{n}\right)\right| \leq n^{-1} A(\nu)$ for any $\nu \in \mathcal{V}_{\mathrm{qm}}$. Thus Hölder's inequality with $p=n$ and $q=n /(n-1)$ gives

$$
\begin{gathered}
\int_{B^{\prime}}|\psi|^{2} \exp (-2 u)=\int_{B^{\prime}}\left(\sum_{1}^{k}\left|g_{n l}\right|^{2} \exp (-2 p u)\right)^{1 / p}\left(|\psi|^{2 q}\left(\sum_{1}^{k}\left|g_{n l}\right|^{2}\right)^{-q / p}\right)^{1 / q} \\
\leq k^{1 / p}\left(\int_{B^{\prime}}|\psi|^{2 q}\left(\sum_{1}^{k}\left|g_{n l}\right|^{2}\right)^{-q / p}\right)^{1 / q}=k^{1 / p}\left(\int_{B^{\prime}}|\psi|^{2 q} \exp \left(-2 q u_{n}\right)\right)^{1 / q} .
\end{gathered}
$$

For any quasimonomial valuation $\nu$, we have

$$
\left|\chi_{u, \psi}(\nu)-\chi_{q u_{n}, \psi^{q}}(\nu)\right|=\left|\frac{\nu(u)}{\nu(\psi)+A(\nu)}-\frac{q \nu\left(u_{n}\right)}{q \nu(\psi)+A(\nu)}\right| \lesssim \frac{1}{n} .
$$

By assumption $\sup \chi_{u, \psi}<1$; hence $\sup \chi_{q u_{n}, \psi^{q}}<1$ for large $n$. By the preceding argument, $|\psi|^{2 q} \exp \left(-2 q u_{n}\right)$ is locally integrable, thus so is $|\psi|^{2} \exp (-2 u)$. 


\section{Singularity EXPOnEnts AND Kiselman NUMBERS}

We now apply our machinery to study the singularities of psh functions.

Theorem 4.1 immediately implies that the complex singularity exponent $c(u)$ and Arnold multiplicity $\lambda(u)$ of a psh function $u$ equal those of its tree transform $g_{u}$ as defined by (2.2). In view of Remark 2.3 and Lemma 2.11 we then have

Corollary 5.1. For any psh function $u$ we have

$$
\lambda(u)=\sup _{\nu \in \mathcal{V}_{\mathrm{qm}}} \frac{\nu(u)}{A(\nu)} \quad \text { and } \quad c(u)=\inf _{\nu \in \mathcal{V}_{\mathrm{qm}}} \frac{A(\nu)}{\nu(u)},
$$

and the supremum and infimum are attained at a valuation of multiplicity one.

Further, if $c=c(u)$, then $\exp (-2 c u)$ is not locally integrable at the origin. In fact, $\operatorname{Vol}\{u<\log r\} \gtrsim r^{2 c(u)}$ as $r \rightarrow 0$.

Remark 5.2. This corollary contains an affirmative answer to the openness conjecture for psh functions in dimension 2; see [DK, Remarks 5.3 and 4.4].

Example 5.3. If $\psi=y^{n}+x^{m}$ with $m<n, m, n$ relatively prime, and $u=\log |\psi|$, then the supremum in (5.1) must be attained at a valuation of the form $\nu_{y, t}, 1 \leq$ $t \leq \infty$. Thus $\lambda(\log |\psi|)=\sup _{t} \max \{n t, m\} /(1+t)=n m /(n+m)$.

Let us rephrase Corollary 5.1 in terms of Kiselman numbers, using Section 1.4.1 If $m(\nu)=1$, then $\nu=\nu_{y, t}$ where $(x, y)$ are local coordinates and $t \geq 1$. Moreover, $\nu(u)=\nu_{t, 1}^{x, y}(u)$ and $A(\nu)=1+t$. Hence $\nu(u) / A(\nu)=\nu_{t /(1+t), 1 /(1+t)}^{x, y}(u)$.

Corollary 5.4. The Arnold multiplicity of $u$ is the supremum of all Kiselman numbers $\nu_{a, b}^{x, y}(u)$ over all choices of local coordinates $(x, y)$ and all choices of weights $(a, b)$ with $a+b=1$.

By fixing the local coordinates $(x, y)$ and letting the weights vary we obtain a lower bound for the Arnold multiplicity proved by Kiselman [Ki2] (in any dimension). We can also bound it in terms of the Lelong number $\nu^{\mathrm{L}}(u)$ :

Corollary 5.5. The Arnold multiplicity $\lambda(u)$ of u satisfies

(i) $\frac{1}{2} \nu^{\mathrm{L}}(u) \leq \lambda(u) \leq \nu^{\mathrm{L}}(u)$;

(ii) $\lambda(u)=\nu^{\mathrm{L}}(u)$ iff $d d^{c} u$ is the current of integration on a smooth curve plus a current with zero Lelong number;

(iii) if $\lambda(u)=\frac{1}{2} \nu^{\mathrm{L}}(u)$, then the Lelong number of the strict transform of $u$ under a single blowup $\pi$ of the origin is at most $\frac{1}{2} \nu^{\mathrm{L}}(u)$ at any point on the exceptional divisor $\pi^{-1}(0)$.

Remark 5.6. The bounds in (i) are due (in any dimension) to Skoda [S]. The characterization in (ii) sharpens a recent result by Blel and Mimouni BM] (see also [Mi]), who proved that if $u$ has Lelong number one, then $\exp (-2 u)$ is locally integrable unless $d d^{c} u$ puts mass on an analytic curve. The implication in (iii) seems to be new.

Remark 5.7. The converse to (iii) is false, as is shown by the example

$$
u=\log \max \left\{|y|,|x|^{1+\varepsilon}\right\}
$$

where $0<\varepsilon<1$. Here $\nu^{\mathrm{L}}(u)=1, \lambda(u)=(1+\varepsilon) /(2+\varepsilon)>1 / 2$, but the strict transform of $u$ has Lelong number zero at all points of $\pi^{-1}(0)$, but one, at which the Lelong number is $\varepsilon$. 
Proof of Corollary [5.5. All of this is a consequence of Theorem 4.1 and Proposition [2.7. Indeed (i) follows immediately, as does (iii) in view of Section 1.4.3 As for (ii) we get that the tree measure $\rho_{u}$ is a point mass at a curve valuation, which must be associated to a smooth analytic curve $\{\phi=0\}$. Siu's Theorem now yields $u=\log |\phi|+u^{\prime}$ with $u^{\prime}$ psh. Clearly $u^{\prime}$ has zero Lelong number.

\section{The ASCENDING CHAin CONDITION}

We now wish to give a new proof of a result describing the structure of the set of complex singularity exponents of holomorphic functions.

As our approach is algebraic, we work in the general case; that is, $R$ is an equicharacteristic zero, two-dimensional, regular local ring with algebraically closed residue field. If $\psi \in R$, let $c(\psi)=c\left(g_{\psi}\right)$ and $\lambda(\psi)=c(\psi)^{-1}$ be the singularity exponent and Arnold multiplicity of $\psi$. Here $g_{\psi}$ is the tree potential of $\psi$. Thus $\lambda(\psi)=\sup _{\mathcal{V}_{\mathrm{qm}}} \chi_{\psi}(\nu)$, where $\chi_{\psi}(\nu)=\nu(\psi) / A(\nu)$. In the analytic case $R=\mathcal{O}_{0}$ we have $c(\psi):=c(\log |\psi|)$ and $\lambda(\psi)=\lambda(\log |\psi|)$.

To motivate the result, first consider $\psi \in \mathfrak{m}$ irreducible and let $\left(\nu_{i}\right)_{0}^{g}$ be the approximating sequence of $\nu_{\psi}$. It follows from Corollary 5.1 that the supremum of $\chi_{\psi}$ is attained at $\nu_{1}$. This gives $c(\psi)=\frac{1}{\nu_{1}(\psi)}+\frac{1}{m(\psi)}$. By Section 1.2.7 $\nu_{1}(\psi)$ is an integer, so that $c(\psi) \in \frac{1}{\mathbf{N}^{*}}+\frac{1}{\mathbf{N}^{*}}$, a result that seems to have first been proved by Igusa [I]; see also [ $\mathrm{Ku}]$. When $\psi$ is no longer assumed to be irreducible, $c(\psi) \notin \frac{1}{\mathbf{N}^{*}}+\frac{1}{\mathbf{N}^{*}}$ in general (see [PS] for a counterexample). Nevertheless we have

Theorem 6.1 ([S, 1992], [Ku, 1999], [PS, 2000]). The set $\mathbf{c}=\{c(\psi): \psi \in \mathfrak{m}\}$ satisfies the ascending chain condition (ACC): any increasing sequence in $\mathbf{c}$ is eventually stationary.

Further, the limit points of $\mathbf{c}$ are 0 and the rational numbers $1 /$ a for $a \geq 1$.

Proof. We will show that $\boldsymbol{\lambda}:=\{\lambda(\psi) ; \psi \in \mathfrak{m}\}$ satisfies the decreasing chain condition (DCC) and that its limit points are $\mathbf{N} \cup\{\infty\}$. Our proof mainly consists of a careful study of the functions $\chi_{\psi}$ on $\mathcal{V}_{\mathrm{qm}}$, for $\psi \in \mathfrak{m}$.

Fix $\psi$ and let $\psi=\prod \phi_{k}^{a_{k}}$ be the decomposition into irreducible factors. For any $k$ we define the valuation $\nu_{k}=\max \left\{\nu \in \mathcal{V}: m(\nu)=1, \nu \leq \nu_{\phi_{k}}\right\}$. This is either $\nu_{\phi_{k}}$ or a divisorial valuation. Write $\nu_{k l}=\nu_{k} \wedge \nu_{l}$ and $\alpha_{k l}=\alpha\left(\nu_{k l}\right)$.

Fact 1. For all $k, l$, either $\alpha_{k l} m_{k}$ or $\alpha_{k l} m_{l}$ is an integer where $m_{k}=m\left(\phi_{k}\right)$.

We first note that $m_{k} \alpha_{k}$ is always an integer (if $\alpha_{k}<\infty$ ). For this we refer repeatedly to Section 1.2.7 As $\nu_{\phi_{k}}>\nu_{k}$, the generic multiplicity $b_{k}$ of $\nu_{k}$ divides $m_{k}$. Thus $\alpha\left(\nu_{k}\right) b\left(\nu_{k}\right)=\alpha_{k} b_{k} \in \mathbf{N}$; hence $\alpha_{k} m_{k} \in \mathbf{N}$. This shows Fact 1 when $\nu_{k l}=\nu_{k}$ or $\nu_{l}$. If this is not the case, then $\nu_{k l}<\nu_{k}, \nu_{k l}<\nu_{l}$. As $m\left(\nu_{k}\right)=m\left(\nu_{l}\right)=1$, $b\left(\nu_{k l}\right)=1$ and again $\alpha_{k l} \in \mathbf{N}$.

Fact 2. The supremum of $\chi_{\psi}$ is attained at a valuation $\nu_{k l}$ for some $k, l$.

After unwinding definitions, Lemma 2.11 (i) and (ii) imply that the supremum of $\chi_{\psi}$ is attained either at $\nu_{\mathfrak{m}}$ or at one of the $\nu_{k l}$. If $\nu_{k l}=\nu_{\mathfrak{m}}$ for some $k$ and $l$, the proof is complete, so assume that $\nu_{k l}>\nu_{\mathfrak{m}}$ for all $k, l$. Then there exists a unique tangent vector $\vec{v}$ at $\nu_{\mathfrak{m}}$ such that $\nu_{k l} \in U(\vec{v})$ for all $k, l$. It is then straightforward to verify that $D_{\vec{v}} \chi_{\psi}>0$. Hence the maximum is not attained at $\nu_{\mathfrak{m}}$ and we are done.

Fact 3. For any $k, l$, we have $\nu_{k l}(\psi) \in \frac{\mathbf{N}^{*}}{m_{k}} \cup \frac{\mathbf{N}^{*}}{m_{l}}$. 
If $\nu_{\phi_{i}} \geq \nu_{k l}$, we have $\nu_{k l}\left(\phi_{i}\right)=\alpha_{k l} m_{i}$, which belongs to $\mathbf{N}^{*} / m_{k} \cup \mathbf{N}^{*} / m_{l}$ by Fact 1. If $\nu_{\phi_{i}} \geq \nu_{k l}$, then $\nu_{k l}\left(\phi_{i}\right)=\nu_{i k}\left(\phi_{i}\right)$. Then either $\nu_{i k}=\nu_{i}$, and $\nu_{k l}\left(\phi_{i}\right) \in \mathbf{N}^{*}$; or $\nu_{i k}<\nu_{i}, \nu_{i k}<\nu_{k l}$ and $\alpha_{i k} \in \mathbf{N}^{*}$ so that $\nu_{k l}\left(\phi_{i}\right) \in \mathbf{N}^{*}$.

Fact 4. Suppose $\lambda(\psi)=\chi_{\psi}\left(\nu_{k l}\right)$. Set $I=\left\{i: \nu_{\phi_{i}} \geq \nu_{k l}\right\}$ and let $J$ be its complement. Then

$$
\sum_{J} a_{j} \nu_{k l}\left(\phi_{j}\right) \leq \sum_{I} a_{i} m_{i}
$$

This inequality follows from the assumption $\lambda(\psi)=\chi_{\psi}\left(\nu_{k l}\right)$. For any $t$ in an interval $T=\left(\alpha_{k l}-\eta, \alpha_{k l}\right)$ for $\eta$ sufficiently small, we have

$$
\chi\left(\nu_{\psi_{i}, t}\right)=\frac{t\left(\sum_{I} a_{i} m_{i}\right)+\sum_{J} a_{j} \nu_{k l}\left(\phi_{j}\right)}{1+t} .
$$

As the supremum of $\chi$ is attained when $t=\alpha_{k l}$, we infer that $\chi^{\prime} \geq 0$ on $T$. A direct computation then shows (6.1).

We are now able to prove the theorem. First note that it suffices to prove that $\boldsymbol{\lambda} \cap(0, C)$ satisfies the DCC for any $C>0$. So pick a sequence $\psi_{n} \in \mathfrak{m}$ such that $\lambda\left(\psi_{n}\right)$ is decreasing and bounded from above by $C$. Since $\lambda(\psi)=\sup \nu(\psi) / A(\nu) \geq$ $m(\psi) / 2$, we can bound the multiplicity $m\left(\psi_{n}\right) \leq 2 C$. For any $n$, we introduce $\phi_{k}^{n}$, $\alpha_{k l}^{n}, I^{n}, J^{n}$ as above. To simplify notation we drop the superscript $n$. We also denote by $\mathcal{N}=\left\{\frac{p}{q}: p, q \in \mathbf{N}^{*}, q \leq 2 C\right\}$. This is a discrete semigroup. Fact 2 implies that we can write

$$
\begin{aligned}
\lambda\left(\psi_{n}\right) & =\frac{\left(\sum_{I} a_{i} m_{i}\right) \alpha_{k l}+\sum_{J} a_{j} \nu_{k l}\left(\phi_{j}\right)}{1+\alpha_{k l}} \\
& =\left(\sum_{I} a_{i} m_{i}\right)-\frac{1}{1+\alpha_{k l}}\left[\sum_{I} a_{i} m_{i}-\sum_{J} a_{j} \nu_{k l}\left(\phi_{j}\right)\right] .
\end{aligned}
$$

We have $\sum_{I} a_{i} m_{i} \leq m\left(\psi_{n}\right) \leq 2 C$, so we may assume that $\sum_{I} a_{i} m_{i}$ is constant, say equal to $D$, for all $n$. Fact 3 implies that $\sum_{J} a_{j} \nu_{k l}\left(\phi_{j}\right)$ belongs to the discrete set $\mathcal{N}$. It is also bounded by $D$ by Fact 4 , so we may assume it is constant. Thus $\lambda\left(\psi_{n}\right)=D-\frac{E}{1+\alpha_{k l}}$ for some $E$. Now $\lambda\left(\psi_{n}\right)$ is increasing; hence $\alpha_{k l}$ is decreasing. Finally Fact 1 shows that $\alpha_{k l} \in \mathcal{N}$ and therefore is constant for large $n$. This proves that $\boldsymbol{\lambda}$ satisfies the DCC.

In order to find the limit points of $\boldsymbol{\lambda}$, suppose $\lambda\left(\psi_{n}\right) \rightarrow \lambda \in \boldsymbol{\lambda}, \lambda \neq \infty$. By extracting a subsequence and using the DCC we may assume that $\lambda\left(\psi_{n}\right)$ is increasing. The argument above applies again: for $n$ large, we have $\lambda\left(\psi_{n}\right)=D-E\left(1+\alpha_{k l}\right)^{-1}$ for some constants $D, E>0$, where $D$ is an integer. If $\lambda\left(\psi_{n}\right)$ is not stationary, $\alpha_{k l}$ increases to infinity, so that $\lambda=D \in \mathbf{N}$. This shows that the limit points of $\boldsymbol{\lambda}$ are included in $\mathbf{N} \cup\{\infty\}$. Conversely, we have $\lambda\left(y^{m}+x^{n}\right)=m n /(m+n)$. Letting $m \rightarrow \infty$ while keeping $n$ constant gives $n \in \boldsymbol{\lambda}$. Letting $m, n \rightarrow \infty$ gives $\infty \in \boldsymbol{\lambda}$. This concludes the proof.

\section{IDEAls AS MUltiplier IDEALS}

Proposition 2.5 asserts that the multiplier ideal associated to any tree potential is integrally closed. Our aim is to prove the converse statement, thereby giving a new proof of a theorem by Lipman and Watanabe [LW]. 
Theorem 7.1. If $J$ is an integrally closed ideal of $R$, then $J=\mathcal{J}(g)$ for some tree potential $g$.

Remark 7.2. Our proof is constructive and generates a tree potential of the form $g=c g_{I}$, where $I$ is an integrally closed ideal and $c>0$. The choices of $I$ and $c$ are not unique.

Remark 7.3. Lipman and Watanabe work on more general rings. They also prove that if $J=I_{\nu}$ with $\nu$ divisorial, then there exists an ideal $I$ with $\mathcal{J}(I)=J$ iff $b(\nu)=1$. More generally it would be interesting to know what integrally closed ideals are of the form $\mathcal{J}(I)$ for an ideal $I$.

Proof. Any integrally closed ideal in $R$ is the product of a principal ideal and a primary ideal. In view of Proposition 2.10 we may hence assume that $J$ is primary.

Let us recall some notation from the analysis of tree transforms of ideals in Section 1.3. The tree measure of $J$ on $\mathcal{V}$ is of the form $\rho_{J}=\sum_{1}^{r} n_{i} b_{i} \nu_{i}$. Here $n_{i} \in \mathbf{N}$ and $\nu_{i}$ is divisorial with generic multiplicity $b_{i}$. Write $\alpha_{i}=\alpha\left(\nu_{i}\right)$ and $A_{i}=A\left(\nu_{i}\right)$. Set $m=m(J)=\sum_{i} n_{i} b_{i}$ and let $\mathcal{T}_{J}$ be the support of the tree potential $g_{J}$, i.e., the smallest subtree containing $\nu_{\mathfrak{m}}$ and all the $\nu_{i}$.

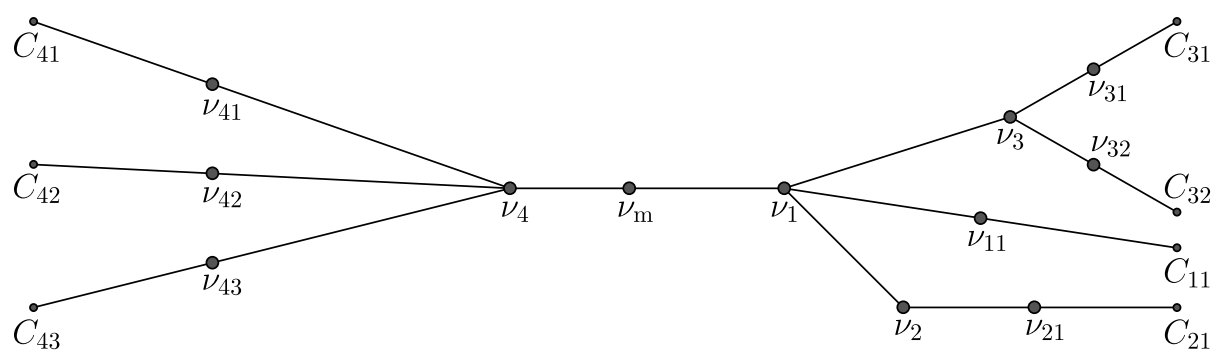

Figure 3. Construction of a tree potential with a predetermined multiplier ideal $I$. The Zariski factorization of $I$ is $I=I_{1} I_{2} I_{3}^{2} I_{4}^{3}$ and $\nu_{i}$ is the Rees valuation of $I_{i}, 1 \leq i \leq 4$. The curves $C_{i j}$ are chosen as curvettes of $\nu_{i}$ and the valuations $\nu_{i j}$ are well chosen on the segments $\left[\nu_{i}, \nu_{C_{i j}}\right]$. See the proof of Theorem 7.1.

Pick $\psi_{i j} \in \mathfrak{m}$ irreducible for $1 \leq i \leq r, 1 \leq j \leq n_{i}$ such that $m\left(\psi_{i j}\right)=b_{i}$, $\nu_{\psi_{i j}}>\nu_{i}$ and the $\nu_{\psi_{i j}}$ represent $n_{i}$ distinct tangent vectors at $\nu_{i}$. The curves $C_{i j}:=\left\{\psi_{i j}=0\right\}$ are then curvettes of $\nu_{i}$. Define $\nu_{i j}$ to be the unique quasimonomial valuation in $] \nu_{i}, \nu_{\psi_{i j}}$ [ whose thinness $A_{i j}=A\left(\nu_{i j}\right)$ is given by $A_{i j}-A_{i}=R_{i}$ for a constant $R_{i}>0$ depending only on $i$ to be fixed shortly. See Figure 3 Write $\alpha_{i j}=\alpha\left(\nu_{i j}\right)$ so that $b_{i}\left(\alpha_{i j}-\alpha_{i}\right)=R_{i}$. For a given $\varepsilon>0$, we define the tree potential $g$ by

$$
\Delta g=(1+\varepsilon) \sum_{i \leq r, j \leq n_{i}} b_{i} \nu_{i j}
$$

Let $\mathcal{T}_{g}$ be the support of $g$ (i.e., $\mathcal{T}_{J}$ with the segments $\left[\nu_{i}, \nu_{i j}\right]$ attached) and let $c(J)=\inf _{\nu} A(\nu) / \nu(J)$ be the complex singularity exponent of the ideal $J$ (see Corollary 5.1). In the sequel, we fix $\varepsilon<m(J)^{-1}$. With this choice, we now show that we can pick $R_{i}>0$, for $i \leq r$, so that

$$
\nu_{i}(J)+A_{i j}-b_{i}^{-1}<g\left(\nu_{i j}\right)<\nu_{i}(J)+A_{i j} .
$$


To see this, notice that

$$
g\left(\nu_{i j}\right)=(1+\varepsilon)\left(\nu_{i}(J)+R_{i}\right) .
$$

Hence $g\left(\nu_{i j}\right)-\nu_{i}(J)-A_{i j}=\varepsilon \nu_{i}(J)-A_{i}+\varepsilon R_{i}=: S_{i}$. We want to pick $R_{i}>0$ so that $S_{i} \in\left(-b_{i}^{-1}, 0\right)$. Clearly $S_{i}$ is an affine function of $R_{i}$ and $S_{i} \rightarrow \infty$ as $R_{i} \rightarrow \infty$. Hence it suffices to show that $S_{i}<0$ when $R_{i}=0$; i.e., that $\varepsilon \nu_{i}(J)<A_{i}$. But the tree potential properties of $g_{J}$ give $\nu_{i}(J) \leq m \alpha_{i}$. Hence $\varepsilon \nu_{i}(J)<\alpha_{i}<A_{i}$.

Finally, we note that $g$ is proportional to the tree transform of some integrally closed ideal. Indeed multiplying $\sum b_{i} \nu_{i j}$ by the least common multiple of all the generic multiplicities $b\left(\nu_{i j}\right)$ we obtain the tree measure $\rho_{I}$ of some ideal $I$. We can thus write $g=c g_{I}$ for some $c>0$ and some integrally closed ideal $I$.

We claim that $\mathcal{J}(g)=J$. Let us first prove that $\mathcal{J}(g) \supset J$. By Proposition 2.4 (ii) it suffices to show that $\nu(J)+A(\nu)>g(\nu)$ for $\nu \in \mathcal{T}_{J}$ and $\nu=\nu_{i j}$. The latter case is taken care of by (3.1). As for the former, if $\nu \in \mathcal{T}_{J}$, then $g(\nu)=(1+\varepsilon) \nu(J)$. Moreover, $\nu(J) \leq m(J) \alpha(\nu)<m(J) A(\nu)$, so

$$
\frac{g(\nu)}{\nu(J)+A(\nu)}=\frac{1+\varepsilon}{1+A(\nu) / \nu(J)}<\frac{1+\varepsilon}{1+m(J)^{-1}}<1 .
$$

We now complete the proof by proving that $\mathcal{J}(g) \subset J$. Fix $\psi \in \mathcal{J}(g)$. Then

$$
\nu(\phi)>g(\nu)-A(\nu)
$$

for all $\nu \in \mathcal{V}_{\mathrm{qm}}$. By Section 1.3 we must show that $\nu_{i}(\phi) \geq \nu_{i}(J)$ for all $i \leq r$.

For $i \leq r$ and any $j \leq n_{i},(7.1)$ and (7.3) imply that

$$
\nu_{i j}(\phi)>g\left(\nu_{i j}\right)-A_{i j}>\nu_{i}(J)-b_{i}^{-1} .
$$

We let $\mathcal{N} \subset\{1, \ldots, r\}$ be the set of $i$ for which there exists $j$ such that $\phi$ has no irreducible factor $\tilde{\phi}$ with $\nu_{\tilde{\phi}} \wedge \nu_{i j}>\nu_{i}$. After relabeling we have $\mathcal{N}=\{1, \ldots, k\}$.

For $1 \leq i \leq k$, we infer immediately that $\nu_{i}(\phi)=\nu_{i j}(\phi)>\nu_{i}(J)-b_{i}^{-1}$. Since $\nu_{i}$ is divisorial we have $\nu_{i}(\phi) \in b_{i}^{-1} \mathbf{N}$ (see Section 1.2.9); hence $\nu_{i}(\phi) \geq \nu_{i}(J)$.

If $k=r$, the proof is complete. Thus assume $k<r$ and pick $i$ with $k<i \leq r$. For any $j \leq n_{i}, \phi$ admits an irreducible factor $\phi_{i j}$ with $\nu_{\phi_{i j}} \wedge \nu_{i j}>\nu_{i}$. The multiplicity of $\phi_{i j}$ is necessarily a multiple of $b_{i}$, say $p_{i j} b_{i}$.

We now replace $\phi_{i j}$ by $\psi_{i j}^{p_{i j}}$, where $\psi_{i j}$ are defined above. By doing this replacement whenever $k<i \leq r$ and $1 \leq j \leq n_{i}$ we obtain from $\phi$ a new $\hat{\phi} \in \mathfrak{m}$, for which $\nu(\hat{\phi})=\nu(\phi)$ for all $\nu \in \mathcal{T}_{J}$.

We can then write $\hat{\phi}=\phi^{\prime} \prod_{k<i<r} \psi_{i}$ where $\psi_{i}=\prod_{j} \psi_{i j}$ and $\phi^{\prime} \in \mathfrak{m}$. Notice that if $\nu \in \mathcal{T}_{J}$, then $\nu\left(\psi_{i}\right)=b_{i} \nu \cdot \nu_{i}$ and $\nu(J)=\sum_{1}^{r} n_{i} b_{i} \nu \cdot \nu_{i}$. Thus, for $\nu \in \mathcal{T}_{J}$ we have

$$
\nu(\phi)-\nu(J)=\nu(\hat{\phi})-\nu(J)=\nu\left(\phi^{\prime}\right)-\sum_{1}^{k} n_{i} b_{i} \nu \cdot \nu_{i}=\nu\left(\phi^{\prime}\right)-\nu\left(J^{\prime}\right),
$$

where $J^{\prime}$ is the integrally closed ideal associated to the measure $\sum_{1}^{k} n_{i} b_{i} \nu_{i}$ (see Section 1.3). We have seen that $\nu_{i}\left(\phi^{\prime}\right)-\nu_{i}\left(J^{\prime}\right)=\nu_{i}(\phi)-\nu_{i}(J)>-b_{i}^{-1}$ for all $i \leq k$. As above we conclude that in fact $\nu_{i}\left(\phi^{\prime}\right)-\nu_{i}\left(J^{\prime}\right) \geq 0$. As the support of $\rho_{J^{\prime}}$ is included in the $\nu_{i}{ }^{\prime}$ s, we infer $\nu\left(\phi^{\prime}\right) \geq \nu\left(J^{\prime}\right)$ for all $\nu \in \mathcal{V}_{\mathrm{qm}}$. Thus (7.4) gives $\nu(\phi) \geq \nu(J)$ for all $\nu \in \mathcal{T}_{J}$. Hence $\phi \in J$, which concludes the proof. 
Remark 7.4. It follows from the proof that we may in fact choose the numbers $R_{i}$ of the form $R_{i}=n / b_{i}$ for $n \in \mathbf{N}^{*}$. One can then check that our construction coincides with that of Lipman and Watanabe [LW].

\section{EQUisingularity}

In general we may refer to two objects as equisingular if they have the same tree transform. Two ideals are equisingular iff they have the same integral closure. (This is classical, but see [F.J1, Theorem 8.12].) Two psh functions are equisingular iff their pull-backs by any composition of blowups have the same Lelong number at all points on the exceptional divisor; see [F.2, Proposition 6.2].

Our objective now is to show that equisingularity can be detected at the level of multiplier ideals. This is achieved through the following theorem, which allows us to recover a tree potential from the multiplier ideals of all its constant multiples.

Theorem 8.1. Suppose $h$ is a potential. For $t>0$, let $h_{t}$ be the tree transform of the ideal $\mathcal{J}(t h)$. Then $h-t^{-1} A \leq t^{-1} h_{t} \leq h$, where $A$ denotes thinness. In particular $t^{-1} h_{t}$ converges to $h$ in $\mathcal{P}$ as $t \rightarrow \infty$.

Remark 8.2. The sequence $\left(n^{-1} h_{n}\right)_{n=1}^{\infty}$ can be thought of as an analogue for a tree potential of the Demailly approximating sequence of a psh function.

Corollary 8.3. Let $h_{1}, h_{2} \in \mathcal{P}$. Then $h_{1}=h_{2}$ iff $\mathcal{J}\left(t h_{1}\right)=\mathcal{J}\left(t h_{2}\right)$ for all $t \geq 0$.

Using the results of Sections 3 and 4 we immediately infer two corollaries.

Corollary 8.4. Let $I_{1}, I_{2}$ be integrally closed ideals. Then $I_{1}=I_{2}$ iff $\mathcal{J}\left(I_{1}^{n}\right)=$ $\mathcal{J}\left(I_{2}^{n}\right)$ for all $n \in \mathbf{N}^{*}$.

Corollary 8.5. Let $u_{1}, u_{2}$ be psh functions. Then $\mathcal{J}\left(t u_{1}\right)=\mathcal{J}\left(t u_{2}\right)$ for all $t \geq 0$ iff the tree transforms of $u_{1}$ and $u_{2}$ coincide.

Remark 8.6. Corollary 8.5 can be deduced directly from Ohsawa-Takegoshi's theorem. Similarly, Corollary 8.4 is a consequence of Skoda's theorem, which implies $\mathcal{J}\left(I^{n}\right)=I^{n-1} \mathcal{J}(I)$.

Remark 8.7. It is not true that if $I_{\bullet}, J_{\bullet}$ are graded systems of ideals with $\mathcal{J}\left(I_{\bullet}^{c}\right)=$ $\mathcal{J}\left(J_{\bullet}^{c}\right)$ for all $c>0$, then $I_{\bullet}=J_{\bullet}$. See Example 3.11

We give a proof of Theorem 8.1 based on the subadditivity property for multiplier ideals proved in DEL.

Proof of Theorem 8.1. One inequality is elementary: for any $\phi \in \mathcal{J}(t h)$ we have $\nu(\phi)>t h(\nu)-A(\nu)$; hence $t^{-1} h_{t} \geq h-A / t$. The other inequality, on the other hand, is quite deep, but it is an immediate consequence of the following result, whose proof is given below.

Lemma 8.8. For any tree potential $h$, the tree transform of $\mathcal{J}(h)$ is dominated by $h$. In other words, $g_{\mathcal{J}(h)} \leq h$.

Remark 8.9. Explicitly, this lemma asserts that for any tree potential $h$, and any $\nu \in \mathcal{V}_{\mathrm{qm}}$, one can find $\phi \in \mathcal{J}(h)$ so that $\nu(\phi) \leq h(\nu)$. It would be interesting to have a more direct construction of such an element $\phi$. 
Proof of Lemma 8.8. Let $\rho=\rho_{h}$ be the measure associated to $h$. We will gradually build the proof using successively more general measures $\rho$.

First suppose $h=g_{I}$ for an integrally closed ideal $I$. If $\psi \in I$, then $\nu(\psi) \geq \nu(I)$ for all $\nu \in \mathcal{V}$. Hence $\nu(\psi)+A(\nu)>\nu(I)$ for all $\nu \in \mathcal{V}_{\text {qm }}$ and $\rho_{\psi}\{\nu\}+m(\nu)>\nu_{I}\{\nu\}$ for all curve valuations $\nu$. By Proposition 2.4 this implies $\psi \in \mathcal{J}(I)$. Hence $I \subset \mathcal{J}(I)$, so Lemma 8.8 is immediate in this case.

We now consider the more general case $h=c g_{I}$, where $I$ is an integrally closed ideal and $c>0$ is rational. By the subadditivity theorem of DemaillyEin-Lazarsfeld DEL] we have $\mathcal{J}\left(I^{c q}\right) \subset \mathcal{J}\left(I^{c}\right)^{q}$ for any $q$. (Our situation is strictly speaking not contained in [DEL]; a more general statement than what we need can be found in [TW].) Therefore $I^{c q} \subset \mathcal{J}\left(I^{c}\right)^{q}$ if $c q \in \mathbf{N}$. Hence $c q g_{I} \geq q g_{\mathcal{J}\left(I^{c}\right)}$, which gives $h \geq g_{\mathcal{J}(h)}$.

The next more general case is when the support of $\rho$ is included in a finite tree $\mathcal{T}$ whose ends are quasimonomial valuations. We approximate $\rho$ by atomic measures $\rho_{n}=\sum_{i} c_{i}^{n} \nu_{i}^{n}$. We can suppose $\nu_{i}^{n}$ are all divisorial valuations, and $c_{i}^{n}$ are rational numbers. We can also impose that $h_{n}=g_{\rho_{n}}$ decreases towards $g_{\rho}=h$. To do so, note that by linearity, we may take the support of $\rho$ to be included in a segment $T=\left[\nu_{\mathfrak{m}}, \nu_{0}\right]$. The set of divisorial valuations in $T$ coincides with the set of valuations with rational skewness and is hence dense in $T$. We now have to approximate a concave function on $T$ by a decreasing sequence of piecewise linear functions with rational slopes, and whose second derivatives are measures supported on the rationals. This can be done in an elementary way.

By Section 1.3. $q h_{n}$ is the tree transform of an integrally closed ideal $I$ if $q$ is the product of the denominators of $c_{i}^{n}$ with the generic multiplicities of $\nu_{i}^{n}$ over all $i$. By the preceding argument, the tree transform of $\mathcal{J}\left(h_{n}\right)$ is bounded by $h_{n}$. As $h \leq h_{n}$ we infer $g_{\mathcal{J}(h)} \leq g_{\mathcal{J}\left(h_{n}\right)} \leq h_{n}$. We conclude, by letting $n \rightarrow \infty$, that $g_{\mathcal{J}(h)} \leq h$ as desired.

Finally consider a general tree potential $h$. First assume that $\rho\{\nu\}<m(\nu)$ for all curve valuations $\nu$. Since $\rho$ has finite mass we may pick $\varepsilon>0$ such that in fact $\rho\{\nu\}<(1-\varepsilon) m(\nu)$. Define $\mathcal{T}=\{\nu \in \mathcal{V}: \rho\{\mu \geq \nu\} \geq(1-\varepsilon) m(\nu)\}$. By Lemma 2.12, $\mathcal{T}$ is a finite subtree of $\mathcal{V}$ and $\mathcal{J}\left(h_{\mathcal{T}}\right)=\mathcal{J}(h)$. Our choice of $\varepsilon$ means that the ends of $\mathcal{T}$ are quasimonomial. Hence the previous analysis gives $g_{\mathcal{J}\left(h_{\mathcal{T}}\right)} \leq h_{\mathcal{T}}$. Since $h_{\mathcal{T}} \leq h$ this yields $g_{\mathcal{J}(h)} \leq h$.

In the most general case we can decompose $h=g_{\phi}+h^{\prime}$, where $\phi \in R$ (possibly reducible) and $h^{\prime}$ is a tree potential with associated measure satisfying $\rho^{\prime}\{\nu\}<m(\nu)$ for all curve valuations $\nu$. By repeated use of Proposition 2.10 we have $\mathcal{J}(h)=$ $\phi \mathcal{J}\left(h^{\prime}\right)$. Therefore the previous step gives

$$
g_{\mathcal{J}(h)}=g_{\phi}+g_{\mathcal{J}\left(h^{\prime}\right)} \leq g_{\phi}+h^{\prime}=h,
$$

which concludes the proof.

\section{Appendix A. Subadditivity And Skoda's Theorem}

In this appendix we prove two fundamental properties of multiplier ideals associated to tree potentials. The proofs appear here as they rely on the corresponding properties of multiplier ideals of formal powers of ideals. It would be interesting to have direct proofs, not using a reduction to the case of powers of ideals. 
Proof of Proposition 2.8. Write $h=h_{1}+h_{2}$. Let $\rho_{i}=\rho_{h_{i}}$ and $\rho=\rho_{h}=\rho_{1}+\rho_{2}$ be the measures associated to $h_{i}$ and $h$, respectively. As in the proof of Lemma 8.8 we will gradually build the proof using successively more general measures.

First suppose that $h_{i}=c_{i} g_{I_{i}}, i=1,2$, for ideals $I_{1}, I_{2}$ and $c_{1}, c_{2}>0$ rational. By Proposition 3.2 our subadditivity statement translates into $\mathcal{J}\left(I_{1}^{c_{1}} \cdot I_{2}^{c_{2}}\right) \subset \mathcal{J}\left(I_{1}^{c_{1}}\right)$. $\mathcal{J}\left(I_{2}^{c_{2}}\right)$, which holds by DEL] (see the proof of Lemma 8.8).

Next suppose the support of $\rho$ is included in a finite tree $\mathcal{T}$ whose ends are quasimonomial valuations. As in the proof of Lemma 8.8 we approximate $h_{i}$ from above by tree potentials of the form $h_{i}^{n}=c_{i}^{n} g_{I_{i}^{n}}$, where $c_{i}^{n}>0$ are rational and $I_{i}^{n}$ are primary ideals. By semicontinuity (Proposition 2.6) we have $\mathcal{J}\left(h_{i}^{n}\right)=\mathcal{J}\left(h_{i}\right)$ and $\mathcal{J}\left(h_{1}^{n}+h_{2}^{n}\right)=\mathcal{J}(h)$ for large $n$, completing the proof in this case.

Now consider general tree potentials $h_{i}$. First assume that $\rho_{i}\{\nu\}<m(\nu)$ for all curve valuations $\nu, i=1,2$. Since $\rho_{i}$ has finite mass we may pick $\varepsilon>0$ so that in fact $\rho_{i}\{\nu\}<(1-\varepsilon) m(\nu)$. Define $\mathcal{T}_{i}=\left\{\nu \in \mathcal{V}: \rho_{i}\{\mu \geq \nu\}\right\}$. By Lemma2.12, $\mathcal{T}_{i}$ is a finite subtree of $\mathcal{V}$ and $\mathcal{J}\left(\left(h_{i}\right)_{\mathcal{T}_{i}}\right)=\mathcal{J}\left(h_{i}\right)$. Our choice of $\varepsilon$ means that the ends of $\mathcal{T}_{i}$ are quasimonomial. Hence the previous analysis gives

$$
\mathcal{J}\left(h_{1}+h_{2}\right) \subset \mathcal{J}\left(\left(h_{1}\right)_{\mathcal{T}_{1}}+\left(h_{2}\right)_{\mathcal{T}_{2}}\right) \subset \mathcal{J}\left(\left(h_{1}\right)_{\mathcal{T}_{1}}\right) \cdot \mathcal{J}\left(\left(h_{2}\right)_{\mathcal{T}_{2}}\right)=\mathcal{J}\left(h_{1}\right) \cdot \mathcal{J}\left(h_{2}\right) .
$$

In the most general case we can decompose $h_{i}=g_{\phi_{i}}+h_{i}^{\prime}$, where $\phi_{i} \in R$ (possibly reducible) and $h_{i}^{\prime}$ is a tree potential with associated measure satisfying $\rho_{i}^{\prime}\{\nu\}<$ $m(\nu)$ for all curve valuations $\nu$. By repeated use of Proposition 2.10 we have $\mathcal{J}\left(h_{i}\right)=\phi_{i} \mathcal{J}\left(h_{i}^{\prime}\right)$ and $\mathcal{J}(h)=\phi_{1} \phi_{2} \mathcal{J}\left(h_{1}^{\prime}+h_{2}^{\prime}\right)$. Therefore the previous step gives

$$
\mathcal{J}\left(h_{1}+h_{2}\right)=\phi_{1} \phi_{2} \mathcal{J}\left(h_{1}^{\prime}+h_{2}^{\prime}\right) \subset \phi_{1} \phi_{2} \mathcal{J}\left(h_{1}^{\prime}\right) \cdot \mathcal{J}\left(h_{2}^{\prime}\right)=\mathcal{J}\left(h_{1}\right) \cdot \mathcal{J}\left(h_{2}\right),
$$

which completes the proof.

Proof of Proposition 2.9. The proof is based on a reduction to the case $h=c g_{I}$ for an integrally closed ideal $I$ and $c>0$ rational. In the latter case the result is well known (see [La, Theorem 9.6.21]) in view of Proposition 3.2. The reduction goes along exactly the same steps as the proof of subadditivity above. The details are left to the reader.

\section{REFERENCES}

[BM] M. Blel and S.K. Mimouni. Singularités et intégrabilité des fonctions plurisousharmoniques. Ann. Inst. Fourier, to appear.

[D] J.-P. Demailly. Multiplier ideal sheaves and analytic methods in algebraic geometry. In School on Vanishing Theorems and Effective Results in Algebraic Geometry (Trieste 2000), 1-148. ICTP Lect. Notes, vol. 6, Abdus Salam Int. Cent. Theoret. Phys., Trieste, 2001. MR.1919457 (2003f:32020)

[DEL] J.-P. Demailly, L. Ein and R. Lazarsfeld. A subadditivity property of multiplier ideals. Michigan Math. J. 48 (2000), 137-156. MR.1786484(2002a:14016)

[DK] J.-P. Demailly and J. Kollár. Semi-continuity of complex singularity exponents and KählerEinstein metrics on Fano orbifolds. Ann. Sci. École Norm. Sup. (4) 34 (2001), no. 4, 525-556. MR 1852009|(2002e:32032)

[ELS] L. Ein, R. Lazarsfeld and K.E. Smith. Uniform approximation of Abhyankar valuation ideals in smooth function fields. Amer. J. Math. 125 (2003), no. 2, 409-440. MR1963690 (2003m:13004)

[FJ1] C. Favre and M. Jonsson. The valuative tree. Lecture Notes in Mathematics, 1853. Springer-Verlag, Berlin, 2004. MF 2097722

[FJ2] C. Favre and M. Jonsson. Valuative analysis of planar plurisubharmonic functions. Invent. Math., to appear.

[I] J. Igusa. On the first terms of certain asymptotic expansions. Complex analysis and algebraic geometry, 357-368. Iwanami Shoten, Tokyo, 1977. MF 0485881 (58:5680) 
[Ki1] C.-O. Kiselman. Un nombre de Lelong raffiné. Séminaire d'analyse complexe et géométrie 1985-1987, pp. 61-70. Faculté des sciences de Tunis et Faculté des Sciences et techniques de Monastir.

[Ki2] C.-O. Kiselman. Attenuating the singularities of plurisubharmonic functions. Ann. Polon. Math. 60 (1994), no. 2, 173-197. MR1301603 (95i:32024)

[Ku] T. Kuwata. On log canonical thresholds of reducible plane curves. Amer. J. Math. 121 (1999), no. 4, 701-721. MR1704476 (2001g:14047)

[La] R. Lazarsfeld. Positivity in algebraic geometry II. Ergebnisse der Math. und ihrer Grenzgebiete (3), vol. 49. Springer-Verlag, Berlin, 2004. MR2095472

[Li] J. Lipman. Adjoints of ideals in regular local rings. Math. Res. Lett. 1 (1994), no. 6, 739-755. With an appendix by S. C. Cutkosky. MR1306018 (95k:13028)

[LW] J. Lipman and K.-I. Watanabe. Integrally closed ideals in two-dimensional regular local rings are multiplier ideals. Math. Res. Lett. 10 (2003), no. 4, 423-434. MR1995782 (2004m:13059)

[MP] J. McKernan and Y. Prokhorov. Threefold thresholds. Manuscripta Math. 114 (2004), 281-304. MR2075967

[Mi] S. K. Mimouni. Singularités des fonctions plurisousharmoniques et courants de Liouville. Thèse de la faculté des sciences de Monastir, Tunisie, 2001.

[N] A. M. Nadel. Multiplier ideal sheaves and Kähler-Einstein metrics of positive scalar curvature. Ann. of Math. (2) 132 (1990), 549-596. MR1078269 (92d:32038)

[PS] D.-H. Phong and J. Sturm. On a conjecture of Demailly and Kollár. Kodaira's issue. Asian J. Math. 4 (2000), no. 1, 221-226. MR.1803721 (2002b:32043)

[S] V. V. Shokurov. Three-dimensional log perestroikas. Russian Acad. Sci. Izv. Math. 40 (1993), no. 1, 95-202. MR1162635 (93j:14012)

[Sp] M. Spivakovsky. Valuations in function fields of surfaces. Amer. J. Math. 112 (1990), no. 1, 107-156. MR $1037606(91 \mathrm{c}: 14037)$

[S] H. Skoda. Sous-ensembles analytiques d'ordre fini ou infini dans $\mathbf{C}^{n}$. Bull. Soc. Math. France 100 (1972), 353-408. MR0352517 (50:5004)

[TW] S. Takagi and K.-I. Watanabe. When does the subadditivity theorem for multiplier ideals hold? Trans. Amer. Math. Soc. 356 (2004), 3951-3961. MR2058513 (2005d:13016)

CNRS, Institut de Mathématiques, Equipe Géométrie et Dynamique, F-75251 Paris Cedex 05, France

E-mail address: favre@math.jussieu.fr

Department of Mathematics, University of Michigan, Ann Arbor, Michigan 481091109

Current address: Department of Mathematics, Royal Institute of Technology, SE-100 44 Stockholm, Sweden

E-mail address: mattiasj@umich.edu

E-mail address: mattiasj@kth.se 